\title{
Gallic acid attenuates blood-spinal cord barrier disruption by inhibiting Jmjd3 expression and activation after spinal cord injury
}

\author{
Chan Sol Park ${ }^{1}$, Jee Lee ${ }^{2}$, Hae Young $\mathrm{Choi}^{1}$, Kwanghyun $\mathrm{Lee}^{3}$, Yeonju Heo ${ }^{4}$, Bong Gun \\ $\mathrm{Ju}^{3}$, Hea-Young Park-Choo ${ }^{4}$, and Tae Yune ${ }^{2}$ \\ ${ }^{1}$ Age-Related and Brain Diseases Research Center, Kyung Hee University \\ ${ }^{2}$ Kyung Hee University \\ ${ }^{3}$ Department of Life Science, Sogang University \\ ${ }^{4}$ School of Pharmacy, Graduate School of Pharmaceutical Sciences
}

June 26, 2020

\begin{abstract}
Background and Purpose: After spinal cord injury (SCI), blood-spinal cord barrier (BSCB) disruption results in secondary injury including apoptotic cell death of neurons and oligodendrocytes, thereby leads to permanent neurological deficits. Recently, we reported that the histone H3K27me3 demethylase Jmjd3 plays a role in regulating BSCB integrity after SCI. Here, we investigated whether gallic acid (GA), a natural phenolic compound that is known to be anti-inflammatory, regulates Jmjd3 expression and activation, thereby attenuates BSCB disruption following the inflammatory response and improves functional recovery after SCI. Experimental Approach: Rats were contused at T9 and treated with GA (50 mg/kg) via intraperitoneal injection immediately, $6 \mathrm{~h}$ and $12 \mathrm{~h}$ after SCI, and further treated for $7 \mathrm{~d}$ with the same dose once a day. To elucidate the underlying mechanism, we evaluated Jmjd3 activity and expression, and assessed BSCB permeability by Evans blue assay after SCI. Key Results: GA significantly inhibited Jmjd3 expression and activation after injury both in vitro and in vivo. GA also attenuated the expression and activation of matrix metalloprotease-9, which is well known to disrupt the BSCB after SCI. Consistent with these findings, GA attenuated BSCB disruption and reduced the infiltration of neutrophils and macrophages compared with the vehicle control. Finally, GA significantly alleviated apoptotic cell death of neurons and oligodendrocytes and improved behavior functions. Conclusions and Implications: Based on these data, we propose that GA can exert a neuroprotective effect by inhibiting Jmjd3 activity and expression followed the downregulation of matrix metalloprotease-9, eventually attenuating BSCB disruption after SCI.
\end{abstract}

\section{Abbreviation}

SCI, Spinal cord injury; BSCB, Blood-spinal cord barrier; B-BB, Blood-brain barrier; GA, Gallic acid; CNS, Central nervous system; IL-6, Interleukin-6; MMP, Matrix metalloprotease; NYU, New York University; i.p., Intraperitoneal; OGD, Oxygen-glucose deprivation; ChIP, Chromatin immunoprecipitation; MPO, Myeloperoxidase; VNM, Ventral motor neuron; GM, Gray matter; WM, White matter; BBB, Basso-BeattieBresnahan; NF200, 200 kDa neurofilament protein; 5-HT, 5-hydroxytryptamine; TJ, Tight junction; ROS, Reactive oxygen species

\section{Introduction}

As a highly selective permeable vascular endothelial structure, the blood-spinal cord barrier (BSCB) provides a particular environment for the cellular constituents of the spinal cord. Impairment of the BSCB plays an important role in the pathogenesis or development of some pathological conditions of the spinal cord because the BSCB mainly regulates the entry of plasma components and blood cells into the spinal cord (Hawkins \& Davis, 2005). Spinal cord injury (SCI) is one of the most severe traumatic injuries and results in primary 
mechanical injury causing axonal and vascular damage at the lesion site; then, a series of pathological events are initiated in response to the primary injury to further impair the wound site and its surrounding regions (Edwards et al., 2014; Giuliano et al., 1999). When the BSCB integrity is damaged after SCI, blood cells such as neutrophils and macrophages are infiltrated into the spinal cord parenchyma and produce inflammatory mediators such as proinflammatory cytokines, contributing to secondary damage (Abbott et al., 2006; Hausmann, 2003; Hawkins \& Davis, 2005; Zlokovic, 2008). These secondary injuries cause apoptotic cell death of neurons and oligodendrocytes resulting in a perpetual neurological deficiency. Therefore, drugs targeting for the prevention of BSCB disruption should facilitate the restriction of cellular damage and functional recovery after SCI.

Histone modification has emerged as a critical regulator of gene expression and has been known to regulate biological events such as development, metabolism, pathogenesis, and diverse cellular responses (Bannister \& Kouzarides, 2011). Recently, our reports showed that the histone H3K27me3 demethylase Jmjd3 is an important epigenetic factor that regulates the integrity of the blood-brain barrier (B-BB) following central nervous system (CNS) injury including SCI (Lee et al., 2016; Lee et al., 2012c; Na et al., 2017). We also reported that Jmjd3 is abruptly upregulated in the blood vessels of injured spinal cord and Jmjd3 functions as an important epigenetic regulator of interleukin-6 (IL-6) gene activation using an in vitroendothelial cell model of ischemia/reperfusion injury (Lee et al., 2012c). Furthermore, Jmjd3 played a critical role in the regulation of B-BB/BSCB integrity by directly upregulating the matrix metalloprotease (MMP) genes, based on in vitro cellular and in vivo animal models (Lee et al., 2016; Na et al., 2017). Therefore, targeting Jmjd3 should be an effective therapeutic strategy to attenuate secondary events after SCI.

Gallic acid (GA, 3, 4, 5-trihydroxy benzoic acid) is a phenolic compound in plants and is estimated to be a putative active compound in tannin. GA and its derivatives are considered to be major polyphenols in grapes, different berries, mango, areca nut, walnut, green tea, and other fruits including wine (Giftson et al., 2010). The diverse pharmacological properties of GA, including antiallergic, anticancer, antioxidant, anti-inflammatory, and neuroprotective effects have previously been reported (Lu et al., 2006; Nabavi et al., 2012; Patel \& Goyal, 2011; Yang et al., 2015; You et al., 2011). Recently, the neuroprotective effect of GA has been reported in several animal models of CNS disorders, such as depression, seizure, Parkinson's disease, Alzheimer's disease, brain trauma, and SCI (Chhillar \& Dhingra, 2013; Huang et al., 2012; Mansouri et al., 2013a; Mansouri et al., 2013b; Sarkaki et al., 2015; Yang et al., 2015). For example, GA improves cognitive, hippocampal long-term potentiation deficits and brain damage induced by chronic cerebral hypoperfusion in rats (Sarkaki et al., 2014). GA also exhibits anti-depressant-like activity in a mouse model of unpredictable chronic mild stress (Chhillar \& Dhingra, 2013) and improves behavior, brain electrophysiology, and inflammation in a rat model of traumatic brain injury via decreasing cerebral pro-inflammatory cytokines (Sarkaki et al., 2015). In addition, GA mitigates SCI-induced oxidative stress and the inflammatory response by increasing the antioxidant status of cells and decreasing the expression of inflammatory factors (Yang et al., 2015). However, the effect of GA on BSCB impairment after SCI has not been investigated yet. Thus, we examined whether GA regulates Jmjd3-mediated BSCB disruption and thereby improves functional recovery by mitigating the apoptosis of neurons and oligodendrocytes after SCI.

\section{Materials and Methods}

Animal

Adult Sprague Dawley rats (MGI Cat\# 5651135, RRID: MGI:5651135; sex: male; weight: 250-300 g) (Samkako, Osan, Korea) were used in this study. Animals were housed under controlled conditions (20$24^{\circ} \mathrm{C}, 55-65 \%$ humidity, $12 \mathrm{~h}$ light/dark cycle), and have been given free access to water and standard laboratory chow (5L79, PMI Nutrition International, St Louis, MO). All animal experiments were performed in accordance with the Guidelines and Policies for Rodent Survival Surgery provided by the Animal Care Committee of the Kyung Hee University (Permission number: KHUASP(SE)-17-059). Animal studies are reported in compliance with the ARRIVE guidelines and with the recommendations made by the British Journal of Pharmacology (Kilkenny et al., 2010). 


\section{SCI surgery}

SCI was performed using New York University (NYU) impactor in accordance with previously described methods (Lee et al., 2014a). Experimental induction of a contusive SCI in a rat model using the NYU impactor device has been validated as an analog to human SCI. A comparison between the rat model of SCI and human SCI shows functional electrophysiological and morphological evidence of similar patterns recorded in motor evoked potentials and somatosensory evoked potentials as well as high-resolution magnetic resonance imaging (Basso et al., 1995). In brief, rats were anesthetized with chloral hydrate $\left(500 \mathrm{mg}[?] \mathrm{kg}^{-1}\right)$ by intraperitoneal (i.p.) injection and laminectomy was performed at the T9-T10 level, exposing the spinal cord beneath without disrupting the dura. The spinous processes of T8 and T11 were then clamped to stabilize the spine, and the exposed dorsal surface of the cord was subjected to moderate contusion injury $(10 \mathrm{~g} \mathrm{X} 25 \mathrm{~mm})$ at the T9-T10 level using a NYU impactor. Throughout the surgical procedure, body temperature was maintained at $37+-0.5 \mathrm{degC}$ with a heating pad (Biomed S.L., Alicante, Spain). After the injury, the muscles and skin were closed in layers, and rats were placed in a temperature and humiditycontrolled chamber overnight. Postoperatively, rats were received subcutaneously supplemental fluids ( $5 \mathrm{ml}$, lactated ringer) and antibiotics (gentamicin, $5 \mathrm{mg}[?] \mathrm{kg}^{-1}$, intramuscular injection) once daily for $5 \mathrm{~d}$. The bladder was emptied manually three times per day until reflexive bladder emptying was established.

Drug treatment

Animals were randomly divided into three experimental groups (Sham, Vehicle, and GA-treated group) (Fig. 1). GA (Cayman Chemical, Ann Arbor, MI) was dissolved in 0.9\% saline and rats were given GA (50 $\mathrm{mg}[?] \mathrm{kg}^{-1}$ ) immediately after SCI by i.p. injection and then received the same dose of GA at $6 \mathrm{~h}$ and $12 \mathrm{~h}$, and then further treated once a day for $7 \mathrm{~d}$. Based on our preliminary study, we found that a dose of 50 $\mathrm{mg} / \mathrm{kg}$ of GA was an optimal dose for the reduction of BSCB disruption after SCI (Fig. 4D) and thus we used $50 \mathrm{mg} / \mathrm{kg}$ of GA throughout this study. Sham-operated animals received no pharmacological treatment and vehicle group received equivolumetric administration of $0.9 \%$ saline.

Tissue preparation

At indicated time points, rats were anesthetized with chloral hydrate $\left(500 \mathrm{mg}[?] \mathrm{kg}^{-1}\right.$, i.p. $)$ and perfused via cardiac puncture initially with $0.1 \mathrm{M}$ PBS and subsequently with $4 \%$ paraformaldehyde in $0.1 \mathrm{M}$ PBS. A 20-mm spinal cord, centered at the lesion site, was dissected out, post-fixed by immersion in the same fixative (4\% paraformaldehyde) for $5 \mathrm{~h}$ and placed in $30 \%$ sucrose in $0.1 \mathrm{M}$ PBS. The segment was embedded in OCT for frozen sections, and longitudinal or transverse sections were then cut at 10 or $20 \mu \mathrm{m}$ on a cryostat (CM1850; Leica, Wetzlar, Germany). For molecular work, rats were perfused with 0.1 M PBS and the segments of the spinal cord $(10 \mathrm{~mm})$ including the lesion site were isolated and frozen at $-80^{\circ} \mathrm{C}$ until use.

Molecular docking study

The structure used as a template for the structure-based docking study was prepared by using the X-ray structure (PDB code 4ASK) (Kruidenier et al., 2012). All the water molecules and ligand were removed, and the hydrogen atoms were added. The coordinate file for the structure of inhibitor compounds was constructed in Sybyl X-2.1.1 and energetically minimized using a Tripos force field with Gasteiger-Huckel charges. Docking was carried using Surflex-Dock GeomX module interfaced in Sybyl X-2.1.1 by generating protocol using the co-crystallized ligand in the binding site (Jain, 2003). The ligand was docked to the receptor and analyzed with the original ligand bound to the receptor to validate the method. The generated compounds were also docked into the binding site and the results were analyzed and ranked by Total Score $\left(-\log K_{d}\right)$.

In vitro histone methylation assay

In vitro Jmjd3 inhibition assay was modified from Epigenase Jmjd3/UTX demethylase activity/Inhibition assay kit (P-3084, Epigentek, Farmingdale, NY). As briefly described, acid-extracted histones were incubated with a Jmjd3 enzyme (E24026-1, Epigentek) in an assay buffer $(0.05 \%$ Tween-20, $0.05 \%$ BSA, $10 \mu \mathrm{M}$ $\mathrm{NH}_{4} \mathrm{FeSO}_{4}, 200 \mu \mathrm{M}$ ascorbic acid, $2 \mu \mathrm{M} \alpha$-ketoglutaric acid, $25 \mathrm{mM}$ HEPES, pH 7.5) with each inhibitor 
as a proper concentration for $2 \mathrm{~h}$ at 37 . The demethylation reaction was stopped by adding a $5 \mathrm{X}$ SDS sample buffer and boiling for $5 \mathrm{~min}$. Western blotting was carried out using anti-H3K27me3 (Millipore, Cat\# 07-449, RRID: AB_310624).

Endothelial cell culture and oxygen-glucose deprivation (OGD)/reperfusion

A mouse brain endothelial cell line, bEnd.3 (ATCC, Cat\# CRL-2299, RRID: CVCL_0170) was cultured as previously described (Lee et al., 2012b; Lee et al., 2012c). Prior to each experiment, cells were seeded onto 6 -well $\left(5 \times 10^{5}\right.$ cells/well $)$ plates. To achieve OGD, cells were transferred to a humidified anaerobic chamber (APM-30D, Astec, Fukuoka, Japan) under an atmosphere of $0.1 \% \mathrm{O}_{2}, 5 \% \mathrm{CO}_{2}$ balanced with $95 \% \mathrm{~N}_{2}$. The culture medium was replaced three times with deoxygenated and glucose-free DMEM. The cells were treated with GA $(10 \mu \mathrm{M})$ for $30 \mathrm{~min}$ before OGD. At the end of the OGD period, cells were placed under normoxic conditions and the media was quickly replaced with $25 \mathrm{mM}$ glucose containing DMEM. Control cells were cultured in DMEM with $25 \mathrm{mM}$ glucose under normoxia. GA $(10 \mu \mathrm{M})$ was dissolved in $0.9 \%$ saline, which had no effect on cell viability.

Chromatin immunoprecipitation (ChIP)

ChIP assay was performed as previously described (Lee et al., 2016; Lee et al., 2012c). The primary antibodies used in ChIP assay are as follows: Jmjd3 (Abgent, Cat\# AP1022a, RRID: AB_889281), H3k27me3 (Millipore), normal IgG (Santa Cruz Biotechnology, Cat\# sc-2027, RRID: AB_737197). Cells were crosslinked using 1\% formaldehyde in PBS for $15 \mathrm{~min}$ at room temperature. To stop the cross-linking, $1.25 \mathrm{M}$ glycine was added (1\% final concentration). After washing with ice-cold PBS, the cells in SDS lysis buffer (1\% SDS, $10 \mathrm{mM}$ EDTA, $50 \mathrm{mM}$ Tris-HCl, $\mathrm{pH}$ 8.1) was sonicated until the DNA fragments were 300-500 bp in size. The extracts were subsequently centrifuged, and the resulting soluble chromatin solutions were diluted 10 fold with ChIP dilution buffer (0.01\% SDS, $1.1 \%$ Triton X-100, $1.2 \mathrm{mM}$ EDTA, $167 \mathrm{mM} \mathrm{NaCl}, 16.7 \mathrm{mM}$ Tris-HCl, $\mathrm{pH}$ 8.1). The specific antibodies or IgG were added into soluble chromatin solution for overnight at $4{ }^{\circ} \mathrm{C}$ and followed by protein A-sepharose beads (Sigma-Aldrich, St. Louis, MO) for $2 \mathrm{~h}$. The beads were extensively washed with low salt wash buffer (0.1\% SDS, $1 \%$ Triton X-100, 2 mM EDTA, $150 \mathrm{mM} \mathrm{NaCl}, 20$ $\mathrm{mM}$ Tris- $\mathrm{HCl}, \mathrm{pH} 8.1)$, high salt wash buffer (0.1\% SDS, 1\% Triton X-100, $2 \mathrm{mM}$ EDTA, $500 \mathrm{mM} \mathrm{NaCl}, 20$ $\mathrm{mM}$ Tris-HCl, $\mathrm{pH} 8.1), \mathrm{LiCl}$ wash buffer $(0.25 \mathrm{M} \mathrm{LiCl}, 1 \% \mathrm{NP}-40,1 \%$ deoxycholate, $1 \mathrm{mM}$ EDTA, $10 \mathrm{mM}$ Tris-HCl, $\mathrm{pH}$ 8.1), and finally with TE buffer. After elution of the DNA/protein with 1\% SDS, crosslinking was reversed for $6 \mathrm{~h}$ at $65{ }^{\circ} \mathrm{C}$. The DNA was recovered using a QIAquick spin column (Qiagen, Valencia, CA). Real time-PCR was performed with a Stratagene Mx3000P using primers that cover mouse, Mmp-3 (5'TTC CGC CTT TTT TGT TCA-3' and 5'-CCA CTC AAA AAC AGG TCT ATA ATT T-3'), and $M m p-9$ (5'-CCC AGG CTC ATC TTT CCT TCC CC-3' and 5'-CCC ATC CCC ACA CTG TAG GTT C-3'). The relative proportions of immunoprecipitated fragments were determined using the $\Delta \mathrm{Ct}$ comparative method based on the threshold cycle $(\mathrm{Ct})$ value for each PCR reaction and normalized to input genomic DNA.

Evans blue assay

BSCB permeability was investigated with Evans Blue dye extravasation as previously described (Lee et al., 2012a). In brief, $5 \mathrm{ml}$ of $2 \%$ Evans blue dye (Sigma) was administered via i.p. injection at $24 \mathrm{~h}$ after SCI and then perfused with 0.1M PBS at $3 \mathrm{~h}$ later. The spinal cords $(5 \mathrm{~mm})$ including lesion epicenter were homogenized and the fluorescence intensity was measured on a Gemini XPS and EM Microplate Readers (Molecular device, Sunnyvale, CA) at $620 \mathrm{~nm}$ excitation/680 nm emission. The Dye in samples was determined as micrograms per gram of tissue from a standard curve plotted using known amounts of dye.

Immunohistochemistry

Frozen sections were processed for immunohistochemistry with antibodies against myeloperoxidase (MPO; 1:100; Agilent, Cat\# A0398, RRID: AB_2335676), ED-1 (1:1,000; Bio-Rad, Cat\# MCA341R, RRID: AB_2291300), cleaved caspase-3 (1:100; Cell Signaling Technology, Cat\# 9661, RRID: AB_2341188), CC1 (1:100; Abcam,, Cat\# ab16794, RRID: AB_443473), Jmjd3 (1:100; Abcam, Cat\# ab38113, RRID: AB_943898) and RECA1 (1:100; Bio-Rad, Cat\# MCA970GA, RRID: AB_567193) as previously described (Lee et al., 2014b). 
For quantification of MPO or ED-1 intensity, serial transverse sections (20 $\mu \mathrm{m}$ thickness) were collected every $100 \mu \mathrm{m}$ section rostral and caudal 3,000 $\mu \mathrm{m}$ to the lesion site (total 60 sections). Digital images of MPO- or ED-1-stained tissues were obtained and quantified the entire fluorescent intensity of each transverse section above the threshold by using MetaMorph software (Molecular devices) and averaged. The threshold value was at least three times the background and the backgrounds were quantified and normalized to the primary antibody omitted control. Immunostaining control studies were performed by omission of the primary antibodies, by replacement primary antibodies with non-immune, control antibody, and by pre-absorption with an excess $(10 \mu \mathrm{g} / \mathrm{ml})$ of the respective antigens. For double labeling, fluorescein isothiocyanate - or cyanin 3-conjugated secondary antibodies (Jackson ImmunoResearch Labs, Cat\# 111-165-003, RRID: AB_2338000) was used. Also, nuclei were labeled with DAPI according to the protocol of the manufacturer (Thermo Fisher Scientific, Cat\# D3571, RRID: AB_2307445). In all controls, reaction to the substrate was absent if the primary antibody was omitted or if the primary antibody was replaced by a nonimmune control antibody. For quantification of cleaved caspase-3-positive oligodendrocytes (cleaved caspase-3/CC1 double positive), serial transverse sections (10 $\mu \mathrm{m}$ thickness) were collected every $200 \mu \mathrm{m}$ from $4,000 \mu \mathrm{m}$ rostral to $4,000 \mu \mathrm{m}$ caudal to the lesion site (total 40 sections). Cleaved caspase-3-positive oligodendrocytes in the white matter (WM) in each section were counted and averaged. Serial sections were also stained with Cresyl violet acetate for histological analysis.

\section{Cell counting of viable ventral motor neurons (VMNs)}

One day after injury, the number of VMNs was counted and assessed. The criteria for VMN counting was based on the previous report (Lee et al., 2014b). In brief, we determined the cells located in the lower ventral horn and were larger than half of the sampling square $(20 \times 20 \mu \mathrm{m})$ as a VMN. The cells above the line at $150 \mu \mathrm{m}$ ventral from the central canal were excluded. For counting of the total number of VMN, serial transverse sections ( $20 \mu \mathrm{m}$ thickness) were collected every millimeter section rostral and caudal $8 \mathrm{~mm}$ to the lesion site and stained with Cresyl violet acetate. Motor neurons were manually counted from each field and analyzed by MetaMorph software (Molecular Devices).

\section{TUNEL staining}

One and five days after injury, serial spinal cord sections (20 $\mu \mathrm{m}$ thickness) were collected every $200 \mu \mathrm{m}$ and processed for TUNEL staining using an ApopTag in situ kit (Millipore, Cat\# S7100, RRID: AB_2661855), according to the manufacturer's instructions. A DAB substrate kit (Vector Laboratories, Burlingame, CA) was used for peroxidase staining. Control sections were treated similarly, but incubated in the absence of TDT enzyme, dUTP-digoxigenin, or anti-digoxigenin Ab, and positive control sections were incubated in DNase 1. TUNEL-positive cells in the gray matter (GM) at $1 \mathrm{~d}$ (total 40 sections) and in the WM at $5 \mathrm{~d}$ (total 100 sections) after SCI were counted and quantified using a $20 \mathrm{x}$ objective. Only those cells showing morphological features of nuclear condensation and/or compartmentalization in the GM and WM were counted as a TUNEL-positive cell.

\section{RNA isolation and RT-PCR}

Total RNA at the indicated time points was isolated from spinal cord segments $(10 \mathrm{~mm})$, centered at the lesion site by using TRIZOL reagent (Invitrogen) and RT-PCR were performed as previously described (Lee et al., 2012b; Lee et al., 2003; Yune et al., 2007). The primers used for RT-PCR were synthesized by Genotech (Daejeon, Korea), and the sequences of all primers are presented in Table 1. After amplification, PCR products were subjected to a 1.5 or $3 \%$ agarose gel electrophoresis and visualized by ethidium bromide staining. The relative density of bands (relative to sham value) was analyzed by the AlphaImager software (Alpha Innotech Corporation, San Leandro, CA). The expression of GAPDH was used as an internal control. Experiments were repeated three times and the values obtained for the relative intensity were subjected to statistical analysis. The gels shown in figures are representative of results from three separate experiments.

Western blot

Total protein preparation at the indicated time points and Western blot analysis were performed as previ- 
ously described (Lee et al., 2012a). Protein sample (30 $\mu \mathrm{g}$ ) was separated on SDS-PAGE gel electrophoresis and transferred to nitrocellulose membrane (Millipore). The membranes were blocked in $5 \%$ non-fat skim milk or $5 \%$ bovine serum albumin in Tris-buffered saline containing tween-20 (0.1\%), and subsequently incubated with antibodies. The primary antibodies used in Western blot are as follows. Jmjd3 (1:1,000; Abcam), H3K27me3 (1:1,000; Abcam, Cat\# ab6002, RRID:AB_305237), Histon H3 (1:1,000; Cell Signaling Technology, Cat\# 9715, RRID: AB_331563), ED-1 (1:1,000; Bio-Rad), iNOS (1:1,000; BD Biosciences, Cat\# 610333, RRID: AB_397723), COX-2 (1:1,000; Cayman Chemical, Cat\# 160107, RRID: AB_10078833), cleaved caspase-3 (1:1,000; Cell Signaling), ZO-1 (1:1,000; Thermo Fisher Scientific, Cat\# 40-2200, RRID: AB_2533456), and occludin (1:1,000; Thermo Fisher Scientific, Cat\# 40-4700, RRID: AB_2533468). $\beta$ tubulin (1:30,000; Sigma-Aldrich, Cat\# T4026, RRID: AB_477577) was used as a loading control. The primary antibody was detected with a horseradish peroxidase-conjugated secondary antibody (Jackson ImmunoResearch). Immunoreactive bands were visualized by chemiluminescence using Supersignal (Thermo Scientific, Rockford, IL). The densitometric values of the bands on Western blots were obtained by AlphaImager software (Alpha Innotech Corporation) and subjected to statistical analysis. Background in films was subtracted from the optical density measurements.

\section{Gelatin zymography}

The activities of MMP-2 and MMP-9 by gelatin zymography were performed using total protein (50 $\mu \mathrm{g})$ at $1 \mathrm{~d}$ after injury as previously described (Lee et al., 2012b). Total protein was loaded on a Novex $10 \%$ zymogram gel (EC61752; Invitrogen) and separated by electrophoresis. The gel was then incubated with renaturing buffer $(2.5 \%$ Triton $\mathrm{X}-100)$ at room temperature for $30 \mathrm{~min}$ to restore the gelatinolytic activity of the proteins. After incubation with developing buffer $(50 \mathrm{mM}$ Tris- $\mathrm{HCl}, \mathrm{pH} 8.5,0.2 \mathrm{M} \mathrm{NaCl}, 5 \mathrm{mM}$ $\mathrm{CaCl}_{2}, 0.02 \%$ Brii35) at $37^{\circ} \mathrm{C}$ for $24 \mathrm{~h}$, the gel was stained with $0.5 \%$ Coomassie and then destained with $40 \%$ methanol containing 10\% acetic acid until appropriate color contrast was achieved. The clear bands on the zymogram were indicative of gelatinase activity. Quantification of bands was performed by AlphaImager software (Alpha Innotech Corporation). Experiments were repeated three times and the values obtained for the relative intensity were subjected to statistical analysis.

\section{Behavioral tests}

The examination of functional deficits after injury was conducted as previously described (Basso et al., 1995; Lee et al., 2003; Rivlin \& Tator, 1977; Yune et al., 2007). Behavioral analyses were performed by trained investigators who were blind as to the experimental conditions. Open-field locomotion to test hindlimb locomotor function was evaluated by using the Basso-Beattie-Bresnahan (BBB) locomotion scale. BBB is a 22-point scale (scores 0-21) that systematically and logically follows recovery of hindlimb function from a score of 0 , indicative of no observed hindlimb movements, to a score of 21 , representative of a normal ambulating rodent. For the inclined plane test, rats were tested in two positions (right side or left side up) on the testing apparatus. The maximum angle at which a rat could maintain its position for $5 \mathrm{~s}$ without falling was recorded for each position and averaged to obtain a single score for each animal. The ability to control and place the hindlimb precisely was tested on a horizontal grid and the analysis was performed by counting the number of footfall (mistake) in foot placing. For the footprint analysis, both forepaws and hind paws were dipped in red and blue dye (nontoxic) and then walked across a narrow box (1 $\mathrm{m}$ long and $7 \mathrm{~cm}$ wide). The footprints were then scanned, and digitized images were analyzed.

Axon counting and myelin staining

After behavioral tests, rats treated with vehicle and GA were perfused at $35 \mathrm{~d}$ after injury, and frozen sections were prepared as described above. For quantitative analysis of axonal density, serial coronal sections collected every millimeter rostral and caudal $5 \mathrm{~mm}$ to the lesion site were stained with an antibody specific for 200 kDa neurofilament protein (NF200, 1:4,000; Sigma-Aldrich, Cat\# N4142, RRID: AB_477272). Some sections were processed for 5-hydroxytryptamine (5-HT, 1:5,000; ImmunoStar, Cat\# 20080, RRID: AB_572263 N) staining. The ABC method was used to detect labeled cells using a Vectastain kit (Vector Laboratories). Axonal densities were determined within preselected fields $\left(40 \times 40 \mu \mathrm{m}, 1,600 \mu \mathrm{m}^{2}\right)$ at specific sites within the 
ventral and dorsolateral funiculi as previously described (Yune et al., 2007). Axons were manually counted from each field and analyzed by MetaMorph software (Molecular Devices). For myelin staining, selected slides were incubated in 0.1\% Luxol fast blue (Solvent Blue 38; Sigma) in acidified 95\% ethanol overnight at $60^{\circ} \mathrm{C}$. Differentiation was performed with $0.05 \%$ lithium carbonate as previous described (Lee et al., 2018). Digital images of Luxol fast blue-stained tissues were obtained by MetaMorph software (Molecular Devices).

Assessment of lesion volume

The measurement of lesion volume using rats tested for behavioral analyses was performed as previously described (Yune et al., 2008). Serial longitudinal sections $(10 \mu \mathrm{m})$ through the dorsoventral axis of the spinal cord were used to measure the lesion volume. Every $50 \mu \mathrm{m}$ section was stained with Cresyl violet acetate. The lesion volume was determined by measuring the area of cavitation at the injury epicenter using a lowpower $(1.25 \mathrm{x})$ objective and then calculated by mean of a MetaMorph software (Molecular Devices). Areas of each longitudinal level are determined, and the total lesion volume was induced by numerical integration of sequential areas.

Statistical analysis

The biochemical assays and analysis of the results was carried out without knowledge of the treatment groups (blinded). Each experiment involved at least 5 independent samples (equal size) per randomized group, and that statistical analysis was done using these independent values. The statistical analysis was undertaken only for studies where each group size was at least $n=5$. The control and test values in Western blot and RTPCR were normalized to an internal standard (such as $\beta$-Tubulin and GAPDH) to reduce variance. Western blot and RT-PCR were normalized to the mean value of the experimental control group to set $\mathrm{Y}$-axis so the control group value was 1 . The units for these normalized data in the Y-axis were the fold of the control group's mean value. All statistical analyses were performed by SPSS 15.0 (SPSS Science, Chicago, IL). Western blot and RT-PCR presented as the mean \pm SD values and Evans blue, BBB, grid walk and inclined plane test data are presented as the mean $\pm \mathrm{SEM}$. Comparisons between vehicle and GA-treated groups were made by unpaired Student's t test. Multiple comparisons between groups were performed one-way ANOVA. Behavioral scores from BBB and inclined plane test were analyzed by repeated measures ANOVA (time vs treatment). Tukey's multiple comparison was used as Post hoc analysis. For each parameter of the data presented, * indicates $p<0.05$.

\section{Results}

GA inhibits the activation and expression of Jmjd3 after SCI

To determine whether GA inhibits the activity and expression of Jmjd3 and thereby exhibits the neuroprotective effect after SCI, we first examined a structure-based docking study to explore whether GA directly inhibits the H3K27me3 demethylation activity of Jmjd3. A co-crystal structure of GA bound to Jmjd3 revealed the critical interactions within the active site (Fig. 2A). GA binds to Jmjd3 by maintaining interactions with Asn1393A, which is located at the entrance to the binding pocket (Fig. 2A; right), and GA revealed a high total docking score, polar score, and low crash score, indicating the presence of noncovalent interactions, such as hydrogen bond interactions (Total Score: 5.46, Polar Score: 5.45, and Crash Score: -0.87). To confirm the inhibitory effect of GA on Jmjd3 enzymatic activity, we performed an in vitro Jmjd3 inhibition assay by using GSK-J4, an inhibitor of Jmjd3 as a positive control. As expected, the level of H3K27me3 was higher in GSK-J4-treated group than in control group, indicating that GSK-J4 inhibits Jmjd3 activity (Fig. 2B; J4). GA treatment also increased the level of H3K27me3 in a dose-dependent manner. (Fig. 2B; GA). Thus, these results suggest that GA directly binds to the active site of Jmjd3 and inhibits Jmjd3 activity (Fig. 2B).

Next, we examined the effect of GA on Jmjd3 expression by RT-PCR and Western blot analysis. The level of Jmjd $3 \mathrm{mRNA}$ and protein was increased after injury as compared with that of the sham control. Furthermore, SCI-induced increase of Jmjd3 mRNA and protein expression was significantly inhibited by GA treatment at $6 \mathrm{~h}$ and $1 \mathrm{~d}$ after injury as compared with those of the vehicle control (Fig. 3A, B). Because Jmjd3 encodes 
a histone demethylase that specifically mediates the removal of methyl groups from H3K27me3/me, the effect of GA on the level of histone H3K27me3 was then examined. The result showed that the protein level of H3K27me3 was markedly decreased at $8 \mathrm{~h}$ and $1 \mathrm{~d}$ after injury, indicating that the activity of Jmjd3 is increased after SCI. In contrast, the level of H3K27me3 was significantly higher in the GA-treated group than in the vehicle group (Fig. 3C). Furthermore, double immunofluorescence with the endothelial cell marker RECA1 clearly revealed that Jmjd3 expression was upregulated in the blood vessels of injured spinal cord at $1 \mathrm{~d}$ after injury (Fig. 3D; Veh), whereas not observed in the blood vessels of uninjured control spinal cord (Fig. 3D; Sham).

\section{GA prevents BSCB disruption by attenuating MMP-9 expression and activation after SCI}

We next asked whether GA treatment inhibits the expression and activity of MMP-2 and MMP-9, which contribute to the disruption of B-BB/BSCB. As shown in Fig. 4A, the level of $M m p-9$ mRNA was increased after injury compared with that of the sham control. However, the SCI-induced increase in $M m p-9 \mathrm{mRNA}$ expression was significantly inhibited by GA treatment at $6 \mathrm{~h}$ and $1 \mathrm{~d}$ after injury as compared with vehicle control, whereas Mmp-2 mRNA expression was not. By gelatin zymography, both the pro-form MMP-2 and MMP-9 were increased at $1 \mathrm{~d}$ after injury, but the active form was increased only in MMP-9, as in the previous reports (Lee et al., 2014b; Lee et al., 2015; Lee et al., 2018). Furthermore, both pro-form MMP-9 and active-form MMP-9 were significantly inhibited by GA as compared with vehicle control, indicating that GA treatment inhibits MMP-9 activity after SCI (Fig. 4B and 4C).

Next, we determined the effect of GA on the BSCB permeability by an Evans blue assay on the control or GA-treated spinal cord at $1 \mathrm{~d}$ after SCI. As shown in Fig. 4D, the amount of Evans blue dye extravasation was increased after SCI when compared with that of the sham control, which indicates that BSCB disruption was induced after SCI. Furthermore, GA treatment significantly reduced the amount of Evans blue dye extravasation, depending on the dose, after SCI (Fig. 4D and E). We next examined the effect of GA on the tight junction (TJ) protein levels of ZO-1 and occludin at $1 \mathrm{~d}$ and $5 \mathrm{~d}$ after injury. As shown in Fig. 4F, the decrease of the levels of ZO-1 at $1 \mathrm{~d}$ and occludin at $5 \mathrm{~d}$ after SCI was significantly attenuated by GA treatment as compared with vehicle control.

GA also inhibits Jmjd3-mediated Mmp-3 and Mmp-9 gene activation and the loss of TJ protein in bEnd.3 cells upon $O G D /$ reperfusion injury.

Previously, we found that Jmjd3 regulates the expression and activation of MMPs, which are involved in the disruption of TJ integrity in brain microvessel endothelial bEnd.3 cells upon OGD/reperfusion injury (Lee et al., 2012c). Thus, we next examined whether GA suppresses the expression of MMP-3 and MMP-9 by ChIP assay in in vitro OGD/reperfusion model using bEnd. 3 cells, thereby inhibits the loss of TJ molecules. The results show that the gene expression of $M m p-3$ and $M m p-9$ by ChIP assay was significantly increased in bEnd.3 cells that were subjected to $6 \mathrm{~h}$ OGD followed by $1 \mathrm{~h}$ of reperfusion (Fig. 5A, +OGD). In contrast, GA suppressed Jmjd3-mediated $M m p$-3 and $M m p-9$ gene activation in bEnd.3 cells (Fig. 5A, +OGD/GA). By Western blot, the expression of Jmjd3 was also increased in bEnd.3 cells subjected to $6 \mathrm{~h}$ OGD followed by immediate, $1 \mathrm{~h}$, and $3 \mathrm{~h}$ of reperfusion when compared to the control. Moreover, the level of H3k27me3 was significantly reduced after OGD/reperfusion injury (Fig. 5B and C +OGD). However, GA decreased the protein level of Jmjd3 and the level of H3k27me3 was significantly higher in GA-treated group at every time point after OGD/reperfusion injury than in the vehicle control (Fig. 5B and C, +OGD/GA), indicating that GA significantly inhibited the activity of the histone H3K27me3 demethylase Jmjd3 after OGD/reperfusion injury. In addition, the expression of the TJ proteins, ZO-1 and occludin, was also decreased (Fig. 5D and $\mathrm{E}$, +OGD), whereas GA significantly attenuated the decrease in ZO-1 and occludin expression in bEnd.3 cells upon OGD/reperfusion injury (Fig. 5D and E, +OGD/GA).

GA attenuates the infiltration of inflammatory cells and the expression of cytokines and chemokines after $S C I$

Since GA prevented BSCB disruption after SCI, we examined the effect of GA treatment on the infiltration of blood cells by immunohistochemistry using antibodies against MPO and ED-1 and Western blot analysis 
for ED-1. Fig. 6A shows a schematic drawing depicting infiltrated neutrophils and macrophages that were observed both rostral and caudal to the lesion area at $1 \mathrm{~d}$ and $5 \mathrm{~d}$ after injury. Examination of the distribution of MPO-positive neutrophils at $1 \mathrm{~d}$ and ED-1-positive macrophages at $5 \mathrm{~d}$ cells along the length of the lesion site showed that these cells were mainly observed in the dorsal column of injured spinal cord. Furthermore, infiltrated neutrophils and macrophages were observed from the lesion site to $2500 \mu \mathrm{m}$, and the farther away from the lesion site, the smaller the number of cells. However, GA treatment significantly alleviated the infiltration of neutrophils and macrophages into any location when compared with vehicle control. The relative fluorescence intensity analysis shows that GA treatment significantly suppressed the infiltration of neutrophils and macrophages compared with that of the vehicle control (Fig. 6B and C). Western blot analysis also revealed that the SCI-induced increase in ED-1 was significantly reduced by GA treatment at $5 \mathrm{~d}$ after injury compared with that of the vehicle control (Fig. 6D).

Next we determined the effect of GA on the expression of inflammatory mediators and chemokines after SCI by RT-PCR and Western blot. The results show that the increases in $T \nu \varphi-\alpha, I \Lambda-1 \beta$ (at $2 \mathrm{~h}$ ), IL-6, Cox-2 and $i N o s$ (at $6 \mathrm{~h}$ ) mRNA levels after SCI were significantly inhibited by GA treatment (Fig. $6 \mathrm{E}$ and F). In addition, GA significantly inhibited the increases in mRNA levels of $M_{\varsigma} \pi-1, M \imath \pi-1 a, M i \pi-1 \beta, \Gamma \rho-a$ (at $2 \mathrm{~h}$ ) and $M i \pi-2 a($ at $6 \mathrm{~h}$ ) after injury (Fig. 6G and H). The protein levels of COX-2 and iNOS at $1 \mathrm{~d}$ after injury were also significantly alleviated in GA-treated group compared with the vehicle control group (Fig. 6I).

\section{GA inhibits apoptotic cell death of neurons and oligodendrocytes after SCI}

To determine the neuroprotective effect of GA after SCI, we performed Cresyl violet staining and compared the number of motor neurons in ventral horn 1d after injury. As shown in Fig. 7A, a massive loss of VMNs was observed in the lesion area after injury, as reported (Yune et al., 2008), while GA treatment significantly inhibited VMN loss in both rostral and caudal to the lesion epicenter when compared with that of the vehicletreated group (Fig. 7A and B). Next, TUNEL staining showed that TUNEL-positive cells were observed mostly near and within the lesion area in the GM at $1 \mathrm{~d}$ (Fig. 7C) and in the WM at $5 \mathrm{~d}$ (Fig. 7D) mostly outside of the lesion area extending the entire length of the section $(20 \mathrm{~mm})$. Furthermore, GA significantly decreased the number of TUNEL-positive neurons in the GM at $1 \mathrm{~d}$ (Fig. 7C and E) and oligodendrocytes in the WM at $5 \mathrm{~d}$ (Fig. 7D and E) when compared with the vehicle control. Immunostaining with cleaved caspase- 3 antibody also revealed that GA treatment significantly reduced the number of activated caspase3 -positive cells in the WM at $5 \mathrm{~d}$ after injury compared to that of the vehicle control (Fig. $7 \mathrm{~F}$ and $\mathrm{H}$ ). Furthermore, double immunofluorescence staining showed that the cleaved caspase-3-positive cells in the WM at $5 \mathrm{~d}$ after SCI were CC1-positive oligodendrocytes (Fig. 7G). In addition, GA treatment significantly decreased the levels of the cleaved forms of caspase-3 at $5 \mathrm{~d}$ after injury compared to that of the vehicle control (Fig. 7I).

\section{GA improves functional recovery after SCI}

To determine the effect of GA on functional recovery, GA (50 mg[?] $\mathrm{kg}^{-1}$, i.p) was treated immediately, $2 \mathrm{~h}$, and $8 \mathrm{~h}$ after injury and then further treated once a day for $7 \mathrm{~d}$. Functional recovery was evaluated for $28 \mathrm{~d}$ after injury using the BBB rating scale, inclined plane test, grid walk test, and footprint analysis. As shown in Fig. 8A, GA significantly increased hindlimb locomotor function from $14 \mathrm{~d}$ to $28 \mathrm{~d}$ after injury compared with that of the vehicle-treated group. In addition, the ability to control and place the hindlimbs precisely was examined on a horizontal grid at $28 \mathrm{~d}$ after injury. As shown in Fig. 8B, the number of mistakes (footfalls on the grid walk) was significantly lower in the GA-treated group than that observed in the vehicle group. The angle of incline determined at $28 \mathrm{~d}$ after injury was also significantly higher in GA-treated rats from $14 \mathrm{~d}$ to $28 \mathrm{~d}$ compared to that in the vehicle group (Fig. 8C). Finally, footprint analysis showed that fairly consistent forelimb-hindlimb coordination was observed in both vehicle- and GA-treated rats at $35 \mathrm{~d}$ after SCI. However, very little toe dragging was shown in GA-treated group, whereas inconsistent dorsal stepping and extensive drags was observed in vehicle-treated rats, as revealed by ink streaks extending from both hindlimbs (Fig. 8D).

GA alleviates axon and myelin loss and decreases lesion volume after SCI 
To examine the correlation between behavioral data and histological outcomes such as axon loss, myelin loss, and lesion volume, we performed histological analysis of the spinal cord tissues from the animals used for behavioral experiments. First, we performed immunostaining with NF200 and 5-HT antibodies to detect remaining axons and the density of preserved axons. In the sham control, NF200-positive axons in both ventral and dorsolateral funiculi were dense, and axonal packing was uniform (Fig. 9A and B, Sham). However, the axon density was markedly decreased in the injured tissue (Fig. 9A and B, Veh). The number of NF200 positive axons in both ventral and dorsolateral funiculi was significantly higher in the GA-treated group compared to that in the vehicle-treated group (Fig. 9A and B). Moreover, the density of 5-HT serotonergic axons in the ventral horn was higher in the GA-treated group compared to that in the vehicletreated group (Fig. 9C). These results suggest that GA treatment alleviates axon loss after SCI. Next, we measured the extent of myelin loss after injury by Luxol fast blue staining. As shown in Fig. 9D, extensive myelin loss near the lesion area was evident in the vehicle-treated group at $35 \mathrm{~d}$ after injury compared with that of the sham control (Fig. 9D, Veh), whereas GA treatment apparently alleviated myelin loss (Fig. 9D, GA).

To evaluate tissue loss after SCI, serial longitudinal sections were stained with Cresyl violet, and the lesion volume was measured. As shown in Fig. 9E, the total lesion volume was significantly reduced in the GA-treated group at $35 \mathrm{~d}$ after injury compared to that of the vehicle-treated group (Fig. 9E).

\section{Discussion}

In this study, we demonstrated that GA has a neuroprotective effect by inhibiting the expression and activation of Jmjd3, thereby preventing BSCB disruption via down-regulating Jmjd3-mediaed activation of MMP-3 and -9 after injury. GA also alleviated the infiltration of blood cells such as neutrophils and macrophages after injury, thereby decreased the expression of cytokines and chemokines, resulting in reduced inflammatory responses. Furthermore, GA treatment inhibited apoptotic cell death of neurons and oligodendrocytes and improved functional recovery after SCI.

In the present study, GA (50 mg[?] $\mathrm{kg}^{-1}$, i.p.) was administered immediately, $2 \mathrm{~h}$ and $8 \mathrm{~h}$ after injury and then was further injected once a day for $7 \mathrm{~d}$ with a same dose of GA. In previous studies, GA (10 mg[?] $\mathrm{kg}^{-1}$, i.p.) treatment exerted protective effects against SCI-induced oxidative stress (Yang et al., 2015). Oral administration of GA (100 mg[?] $\mathrm{kg}^{-} 1$ ) also improved behavior, brain electrophysiology, and inflammation in a rat model of traumatic brain injury (Sarkaki et al., 2015). In addition, the report by Sun. J et al.(Sun et al., 2017) showed that GA (50 mg[?] $\mathrm{kg}^{-1}, 5$ d, i.v.) exhibited the neuroprotective effect against cerebral ischemia/reperfusion injury. When we tested the efficacy of various concentrations of GA (25, 50 , or $100 \mathrm{mg}[?] \mathrm{kg}^{-1}$, i.p.), $50 \mathrm{mg} / \mathrm{kg}$ of GA was most effective for the reduction in BSCB disruption (See Fig. 4D). Furthermore, neither significant side effects nor increased mortality following GA treatment was observed. Therefore, we suspect that the dosage of GA (50 mg[?] $\mathrm{kg}^{-1}$, i.p.) used in this study is the optimal concentration of GA.

The BSCB, which is a unique barrier between the circulating blood and the CNS, is composed of endothelial cells, astrocyte end-feet, pericytes, the basal lamina, and tight/adherence junction proteins (Hawkins \& Davis, 2005). BSCB breakdown facilitates immune cell infiltration and triggers the posttraumatic inflammatory response (Hausmann, 2003; Lee et al., 2015; Noble et al., 2002). Recently, we reported that the regulation of Jmjd3 may serve as a new therapeutic intervention for preventing BSCB disruption after SCI, which is based on the experimental evidence that Jmjd3-mediated MMP activation is involved in BSCB disruption after SCI (Lee et al., 2014a; Lee et al., 2016). Thus, we showed in this study that GA, a natural compound, significantly inhibits Jmjd3 activity as a Jmjd3 inhibitor and expression after injury. Furthermore, the expression of MMP-3 and MMP-9 by ChIP assay in vitro OGD/reperfusion model using bEnd.3 cells was significantly inhibited by GA. Previously, we also showed that MMP-3 is involved in BSCB disruption after SCI (Lee et al., 2014a; Lee et al., 2016). Thus, these results suggest that the prevention of BSCB disruption by GA may be mediated by inhibiting Jmjd3 activity followed the inhibition of MMP3 and/or MMP-9 activation and expression after SCI. However, the effect of GA on MMP-3 activation and expression after SCI was not examined in this study and further study about this effect will be examined. 
Accumulated studies have shown that GA has an anti-oxidant and anti-inflammatory activities (Huang et al., 2012; Mansouri et al., 2013b; Sarkaki et al., 2015). Reactive oxygen species (ROS) as well as reactive nitrogen species such as nitric oxide and peroxynitrite have been known to induce apoptotic cell death. Additionally, superoxide anion, one of the major ROS has been known to regulate the activation of inflammatory genes such as COX-2 and iNOS (Carroll et al., 2000; Kurtoglu et al., 2014). The report by Wang et al.(Yang et al., 2015) showed that GA exerts neuroprotective effect by mitigating SCI-induced oxidative stress and inflammatory response. Furthermore, ROS has been known to play an important role in the B-BB disruption and endothelial cell permeability (Schreibelt et al., 2007). However, the effect of GA on oxidative stress followed BSCB disruption after SCI was not examined in this study. Therefore, we can't exclude the possibility that the prevention of BSCB disruption after SCI by GA may be mediated through the antioxidant and/or anti-inflammatory effect of GA and further study about this view is needed.

In previous our report, we showed that Jmjd3 up-regulation, in cooperation with NF- $\varkappa \mathrm{B}$ is required for Mmp3 and Mmp-9 gene expressions in injured vascular endothelial cells results in the BSCB disruption after SCI (Lee et al., 2012c). Furthermore, we investigated and confirmed in this study that the neuroprotective effect and anti-inflammatory response of GA was mediated by inhibiting Jmjd3 activation and expression and thereby preventing BSCB disruption after SCI. However, it has also been known that Jmjd3 is involved in several physiological functions, including the inflammatory response and cell death (Tang et al., 2014; Yang et al., 2016). For example, the suppression of Jmjd3 mediates M1 microglial inflammatory responses by inhibiting M2 microglia polarization, leading to dopamine neuron death in 1-methyl-4-phenyl-1, 2, 3, 6-tetrahydropyridine (MPTP)-induced mouse model of Parkinson's disease (Tang et al., 2014). Also, it was reported that Jmjd3 regulates osteoblast apoptosis through targeting anti-apoptotic protein Bcl-2 and pro-apoptotic protein Bim (Yang et al., 2016). In addition, Jmjd3 is known to be up-regulated by many inflammatory mediators and stress inducers through different signaling pathways including STAT signaling. For example, Jmjd3 is activated by STAT-1 and STAT-3 in LPS-treated primary microglia cell and thereby enhances the transcription of crucial inflammatory genes (Przanowski et al., 2014). STAT-3 is also upregulated and plays a critical role in regulating reactive astrogliosis and scar formation after SCI (Herrmann et al., 2008). Thus, we can't rule out the possibility that Jmjd3 may influence on the various pathological events such as apoptotic cell death of neuron and oligodendrocyte, and microglial activation followed inflammatory response after SCI besides the regulation of BSCB integrity. Thus, further study will be needed to elucidate the precise role and underlying mechanisms of Jmjd3 in various pathological processes after SCI.

In summary, our findings showed that the neuroprotective effect of GA after SCI is mediated, in part, by inhibiting the expression and activation of Jmjd3, which thereby prevents BSCB disruption and subsequent blood cell infiltration by inhibiting the activation and expression of MMP-9 and/or MMP-3. Through the elucidation of the neuroprotective effect of GA by inhibiting Jmjd3 expression and activity, our study suggests that GA may be a potentially useful therapeutic agent for traumatic SCI.

\section{Declaration of Transparency and Scientific Rigour}

This Declaration acknowledges that this paper adheres to the principles for transparent reporting and scientific rigour of preclinical research as stated in the BJP guidelines for Design and Analysis, Immunoblotting and Immunochemistry, and Animal Experimentation, and as recommended by funding agencies, publishers and other organisations engaged with supporting research.

\section{Author's contributions}

C.S.P, J.Y.L., and T.Y.Y. designed the research. C.S.P, H.Y.C and J.Y.L. performed the

experiments and analyzed the data. K.L and B.G.J provided the in vitro histone methylation assay and ChIP assay. Y.H. and H.P.C. performed molecular docking study. C.S.P., J.Y.L., and T.Y.Y. wrote the manuscript.

\section{References}


Abbott NJ, Ronnback L, \& Hansson E. (2006). Astrocyte-endothelial interactions at the blood-brain barrier. Nat Rev Neurosci, 7 (1), 41-53.

Bannister AJ, \& Kouzarides T. (2011). Regulation of chromatin by histone modifications. Cell Res, 21 (3), 381-395.

Basso DM, Beattie MS, \& Bresnahan JC. (1995). A sensitive and reliable locomotor rating scale for open field testing in rats. J Neurotrauma, 12 (1), 1-21.

Carroll JE, Hess DC, Howard EF, \& Hill WD. (2000). Is nuclear factor-kappaB a good treatment target in brain ischemia/reperfusion injury? Neuroreport, 11 (9), R1-4.

Chhillar R, \& Dhingra D. (2013). Antidepressant-like activity of gallic acid in mice subjected to unpredictable chronic mild stress. Fundam Clin Pharmacol, 27 (4), 409-418.

Edwards WB, Schnitzer TJ, \& Troy KL. (2014). Bone mineral and stiffness loss at the distal femur and proximal tibia in acute spinal cord injury. Osteoporos Int, 25 (3), 1005-1015.

Giftson JS, Jayanthi S, \& Nalini N. (2010). Chemopreventive efficacy of gallic acid, an antioxidant and anticarcinogenic polyphenol, against 1,2-dimethyl hydrazine induced rat colon carcinogenesis. Invest New Drugs, 28 (3), 251-259.

Giuliano F, Hultling C, El Masry WS, Smith MD, Osterloh IH, Orr M, et al. (1999). Randomized trial of sildenafil for the treatment of erectile dysfunction in spinal cord injury. Sildenafil Study Group. Ann Neurol, $46(1), 15-21$.

Hausmann ON. (2003). Post-traumatic inflammation following spinal cord injury. Spinal Cord, 41 (7), 369-378.

Hawkins BT, \& Davis TP. (2005). The blood-brain barrier/neurovascular unit in health and disease.Pharmacol Rev, 57 (2), 173-185.

Herrmann JE, Imura T, Song B, Qi J, Ao Y, Nguyen TK, et al. (2008). STAT3 is a critical regulator of astrogliosis and scar formation after spinal cord injury. J Neurosci, 28 (28), 7231-7243.

Huang HL, Lin CC, Jeng KC, Yao PW, Chuang LT, Kuo SL, et al. (2012). Fresh green tea and gallic acid ameliorate oxidative stress in kainic acid-induced status epilepticus.J Agric Food Chem, 60 (9), 2328-2336.

Jain AN. (2003). Surflex: fully automatic flexible molecular docking using a molecular similarity-based search engine. J Med Chem, 46 (4), 499-511.

Kilkenny C, Browne W, Cuthill IC, Emerson M, Altman DG, \& Group NCRRGW. (2010). Animal research: reporting in vivo experiments: the ARRIVE guidelines. J Gene Med, 12 (7), 561-563.

Kruidenier L, Chung CW, Cheng Z, Liddle J, Che K, Joberty G, et al. (2012). A selective jumonji H3K27 demethylase inhibitor modulates the proinflammatory macrophage response.Nature, 488 (7411), 404-408.

Kurtoglu T, Basoglu H, Ozkisacik EA, Cetin NK, Tataroglu C, Yenisey C, et al. (2014). Effects of cilostazol on oxidative stress, systemic cytokine release, and spinal cord injury in a rat model of transient aortic occlusion. Ann Vasc Surg, 28 (2), 479-488.

Lee JY, Choi HY, Ahn HJ, Ju BG, \& Yune TY. (2014a). Matrix metalloproteinase-3 promotes early bloodspinal cord barrier disruption and hemorrhage and impairs long-term neurological recovery after spinal cord injury. Am J Pathol, 184 (11), 2985-3000.

Lee JY, Choi HY, Na WH, Ju BG, \& Yune TY. (2014b). Ghrelin inhibits BSCB disruption/hemorrhage by attenuating MMP-9 and SUR1/TrpM4 expression and activation after spinal cord injury. Biochim Biophys Acta, 1842 (12 Pt A), 2403-2412. 
Lee JY, Choi HY, Na WH, Ju BG, \& Yune TY. (2015). 17beta-estradiol inhibits MMP-9 and SUR1/TrpM4 expression and activation and thereby attenuates BSCB disruption/hemorrhage after spinal cord injury in male rats.Endocrinology, 156 (5), 1838-1850.

Lee JY, Choi HY, Park CS, Ju BG, \& Yune TY. (2018). Mithramycin A Improves Functional Recovery by Inhibiting BSCB Disruption and Hemorrhage after Spinal Cord Injury. J Neurotrauma, 35 (3), 508-520.

Lee JY, Kim HS, Choi HY, Oh TH, Ju BG, \& Yune TY. (2012a). Valproic acid attenuates blood-spinal cord barrier disruption by inhibiting matrix metalloprotease-9 activity and improves functional recovery after spinal cord injury. J Neurochem, 121 (5), 818-829.

Lee JY, Kim HS, Choi HY, Oh TH, \& Yune TY. (2012b). Fluoxetine inhibits matrix metalloprotease activation and prevents disruption of blood-spinal cord barrier after spinal cord injury. Brain, 135 (Pt 8), 2375-2389.

Lee JY, Na WH, Choi HY, Lee KH, Ju BG, \& Yune TY. (2016). Jmjd3 mediates blood-spinal cord barrier disruption after spinal cord injury by regulating MMP-3 and MMP-9 expressions. Neurobiol Dis, 95 , 66-81.

Lee K, Na W, Lee JY, Na J, Cho H, Wu H, et al. (2012c). Molecular mechanism of Jmjd3-mediated interleukin-6 gene regulation in endothelial cells underlying spinal cord injury.J Neurochem, 122 (2), 272282.

Lee SM, Yune TY, Kim SJ, Park DW, Lee YK, Kim YC, et al. (2003). Minocycline reduces cell death and improves functional recovery after traumatic spinal cord injury in the rat.J Neurotrauma, 20 (10), 1017-1027.

Lu Z, Nie G, Belton PS, Tang H, \& Zhao B. (2006). Structure-activity relationship analysis of antioxidant ability and neuroprotective effect of gallic acid derivatives. Neurochem Int, 48 (4), 263-274.

Mansouri MT, Farbood Y, Sameri MJ, Sarkaki A, Naghizadeh B, \& Rafeirad M. (2013a). Neuroprotective effects of oral gallic acid against oxidative stress induced by 6-hydroxydopamine in rats. Food Chem, 138 (2-3), 1028-1033.

Mansouri MT, Naghizadeh B, Ghorbanzadeh B, Farbood Y, Sarkaki A, \& Bavarsad K. (2013b). Gallic acid prevents memory deficits and oxidative stress induced by intracerebroventricular injection of streptozotocin in rats.Pharmacol Biochem Behav, 111, 90-96.

Na W, Shin JY, Lee JY, Jeong S, Kim WS, Yune TY, et al. (2017). Dexamethasone suppresses JMJD3 gene activation via a putative negative glucocorticoid response element and maintains integrity of tight junctions in brain microvascular endothelial cells. J Cereb Blood Flow Metab, 37 (12), 3695-3708.

Nabavi SF, Habtemariam S, Jafari M, Sureda A, \& Nabavi SM. (2012). Protective role of gallic acid on sodium fluoride induced oxidative stress in rat brain. Bull Environ Contam Toxicol, 89 (1), 73-77.

Noble LJ, Donovan F, Igarashi T, Goussev S, \& Werb Z. (2002). Matrix metalloproteinases limit functional recovery after spinal cord injury by modulation of early vascular events. J Neurosci, 22 (17), 7526-7535.

Patel SS, \& Goyal RK. (2011). Cardioprotective effects of gallic acid in diabetes-induced myocardial dysfunction in rats. Pharmacognosy Res, 3 (4), 239-245.

Przanowski P, Dabrowski M, Ellert-Miklaszewska A, Kloss M, Mieczkowski J, Kaza B, et al. (2014). The signal transducers Stat1 and Stat3 and their novel target Jmjd3 drive the expression of inflammatory genes in microglia. J Mol Med (Berl), 92 (3), 239-254.

Rivlin AS, \& Tator CH. (1977). Objective clinical assessment of motor function after experimental spinal cord injury in the rat. J Neurosurg, 47 (4), 577-581.

Sarkaki A, Farbood Y, Gharib-Naseri MK, Badavi M, Mansouri MT, Haghparast A, et al. (2015). Gallic acid improved behavior, brain electrophysiology, and inflammation in a rat model of traumatic brain injury. Can J Physiol Pharmacol, 93 (8), 687-694. 
Sarkaki A, Fathimoghaddam H, Mansouri SM, Korrani MS, Saki G, \& Farbood Y. (2014). Gallic acid improves cognitive, hippocampal long-term potentiation deficits and brain damage induced by chronic cerebral hypoperfusion in rats. Pak J Biol Sci, 17 (8), 978-990.

Schreibelt G, Kooij G, Reijerkerk A, van Doorn R, Gringhuis SI, van der Pol S, et al. (2007). Reactive oxygen species alter brain endothelial tight junction dynamics via RhoA, PI3 kinase, and PKB signaling. FASEB J, 21 (13), 3666-3676.

Sun J, Ren DD, Wan JY, Chen C, Chen D, Yang H, et al. (2017). Desensitizing Mitochondrial Permeability Transition by ERK-Cyclophilin D Axis Contributes to the Neuroprotective Effect of Gallic Acid against Cerebral Ischemia/Reperfusion Injury.Front Pharmacol, 8, 184.

Tang Y, Li T, Li J, Yang J, Liu H, Zhang XJ, et al. (2014). Jmjd3 is essential for the epigenetic modulation of microglia phenotypes in the immune pathogenesis of Parkinson's disease. Cell Death Differ, 21 (3), 369-380.

Yang D, Okamura H, Teramachi J, \& Haneji T. (2016). Histone demethylase Jmjd3 regulates osteoblast apoptosis through targeting anti-apoptotic protein Bcl-2 and pro-apoptotic protein Bim. Biochim Biophys Acta, 1863 (4), 650-659.

Yang YH, Wang Z, Zheng J, \& Wang R. (2015). Protective effects of gallic acid against spinal cord injuryinduced oxidative stress. Mol Med Rep, 12 (2), 3017-3024.

You BR, Kim SZ, Kim SH, \& Park WH. (2011). Gallic acid-induced lung cancer cell death is accompanied by ROS increase and glutathione depletion. Mol Cell Biochem, 357 (1-2), 295-303.

Yune TY, Lee JY, Jiang MH, Kim DW, Choi SY, \& Oh TH. (2008). Systemic administration of PEP1-SOD1 fusion protein improves functional recovery by inhibition of neuronal cell death after spinal cord injury. Free Radic Biol Med, 45 (8), 1190-1200.

Yune TY, Lee JY, Jung GY, Kim SJ, Jiang MH, Kim YC, et al. (2007). Minocycline alleviates death of oligodendrocytes by inhibiting pro-nerve growth factor production in microglia after spinal cord injury. $J$ Neurosci, 27 (29), 7751-7761.

Zlokovic BV. (2008). The blood-brain barrier in health and chronic neurodegenerative disorders. Neuron, 57 (2), 178-201.

\section{Figure legends}

Figure 1. Scheme of the in vivo study design. (Experimental 1) Time course of sample preparation. (Experimental 2) Evans blue assay. (Experimental 3) Behavior test. GA (50 mg[?] $\mathrm{kg}^{-1}$, i.p) was injected immediately after SCI and then received the same dose of GA at $6 \mathrm{~h}$ and $12 \mathrm{~h}$, and then further treated once a day for $7 \mathrm{~d}$.

Figure 2. GA directly binds to the active site of Jmjd3 and inhibits Jmjd3 activity. (A) Stereoview of the intermolecular interactions between the bound GA and the Jmjd3 enzyme residues that line the catalytic pocket shown as dashed lines. (B) The lysates from OGD/reperfusion-injured bEnd.3 cells were immunoprecipitated with the anti-Jmjd3, and immunoblotted with the anti-H3K27me3. GSK-J4 was used as a positive control. Immunoblotting was analyzed quantitatively $(\mathrm{n}=5)$. All data represent mean \pm SD. $*$ p $<0.05$ vs. Jmjd3 only.

Figure 3. GA inhibits the activation and expression of Jmjd3 after SCI. Rats were administered immediately with GA (50 mg[?] $\left.\mathrm{kg}^{-1}\right)$ after SCI and spinal cord tissues were isolated at indicated time after injury. (A) The expression level of Jmjd3 was determined by RT-PCR (at $6 \mathrm{~h}$ and $1 \mathrm{~d}$ ) and (B) Western blot (at $8 \mathrm{~h}$ and $1 \mathrm{~d})$ after SCI and each experiment was analyzed quantitatively $(\mathrm{n}=5)$. (C) The activity of Jmjd3 was measured by Western blot for anti-H3K27me3 at $8 \mathrm{~h}$ and $1 \mathrm{~d}$ after SCI and Western blot was quantified (n $=5$ ). All data represent mean $+-\mathrm{SD} .{ }^{*} \mathrm{p}<0.05$ vs. vehicle. (D) Representative fluorescence microscopic photographs showed that Jmjd3-positive blood vessel endothelial cells are positive for RECA1 at $1 \mathrm{~d}$ after injury. Scale bar, $30 \mu \mathrm{m}$. 
Figure 4. GA inhibits the increase of BSCB permeability by suppressing MMP-9 expression and activation after SCI. Rat was administered with GA after SCI and spinal cord tissues from $6 \mathrm{~h}$ and $1 \mathrm{~d}$ were processed for RT-PCR $(\mathrm{n}=5)$ and gelatin zymography $(\mathrm{n}=5)$. (A) RT-PCR for $M m p$-2 and -9 and densitometric analysis of RT-PCR. (B) Gelatin zymography. (C) Densitometric analysis of zymography. All data represent mean \pm SD. ${ }^{*} p<0.05$ vs. vehicle. (D) Representative spinal cord showing Evans blue dye permeabilized into moderately injured spinal cord and $(\mathrm{E})$ quantification of the amount of Evans blue at $1 \mathrm{~d}$ after injury. Data represent as mean \pm SEM. ${ }^{*} p<0.05$ vs. vehicle. (F) The expression levels of TJ molecules were determined by Western blot with anti-ZO-1 (at $1 \mathrm{~d}$ ) and anti-occludin (at $5 \mathrm{~d}$ ) after injury. Western blot was analyzed quantitatively $(\mathrm{n}=5)$. Data represent mean $\pm \mathrm{SD} .{ }^{*} p<0.05$ vs. vehicle.

Figure 5. GA suppresses Jmjd3-mediated MMP-3 and -9 gene activation and inhibits TJs loss in OGDinduced bEnd.3 cells. (A) ChIP assay was performed in bEnd.3 cells subjected to $1 \mathrm{~h}$ of OGD/reperfusion injury using anti-Jmjd3. The occupancy of anti-Jmjd3 at the MMP-3 and MMP-9 promoter region was calculated via quantitative PCR. (B) Western blots for Jmjd3 and H3K27me3 was determined in bEnd.3 cells subjected to OGD/reperfusion injury. (C) Densitometric analysis of Western blot for Jmjd3 (Left) and H3K27me3 (Right) $(\mathrm{n}=5)$. (D) Western blots for ZO-1 and occludin was determined in bEnd.3 cells subjected to OGD/reperfusion injury. (E) Densitometric analysis of Western blots for ZO-1 (Left) and occludin (Right) $(\mathrm{n}=5)$. Data represent mean $\pm \mathrm{SD} .{ }^{*} p<0.05$ vs. +OGD.

Figure 6. GA suppresses Jmjd3-mediated MMP-3 and -9 gene activation and inhibits TJs loss in OGDinduced bEnd.3 cells. (A) Schematic drawings showing infiltrated neutrophils and macrophages positive cells in both the rostral and caudal to the lesion area. MPO-positive neutrophils (green) at $1 \mathrm{~d}$ and ED-1-positive macrophages (red) at $5 \mathrm{~d}$ in the injured spinal cord tissues. (B) Representative photographs showing MPOpositive neutrophils and ED-1-positive macrophages in the dorsal column of injured spinal tissues at 1500 and $2000 \mu \mathrm{m}$ rostral to lesion epicenter. Scale bar, $50 \mu \mathrm{m}$. (C) Relative fluorescent intensity of MPO- and ED-1-positive cells $(\mathrm{n}=5)$. (D) Western blot (upper) and densitometric analysis (bottom) of ED-1 at 5 $\mathrm{d}$ after injury. To determine the expression of cytokines and chemokines, RT-PCR and Western blot were performed at indicated time points after injury $(\mathrm{n}=5)$. (E) RT-PCR and $(\mathrm{F})$ densitometric analysis of cytokines $(I \lambda-1 \beta$ and $T \nu \varphi-a$ (at $2 \mathrm{~h}$ ), Il-6,Cox-2 andiNos (at $6 \mathrm{~h})$ after injury). (G) RT-PCR and (H)

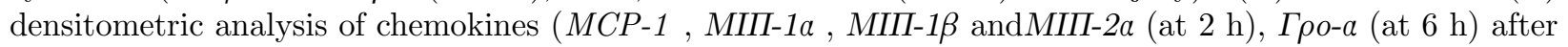
injury and (I) Western blot and densitometirc analysis of inflammatory mediators (iNOS and COX-2 at $1 \mathrm{~d}$ after injury). All data represent mean \pm SD. ${ }^{*} p<0.05$ vs. vehicle.

Figure 7. GA inhibits apoptotic cell death of motor neurons and oligodendrocytes after SCI. (A) Representative Cresyl violet staining showing ventral horn of spinal cord at $3 \mathrm{~mm}$ rostral from lesion site at $1 \mathrm{~d}$. Scale bar, $50 \mu \mathrm{m}$. (B) The spatial pattern of the number of VMN. (C) Representative TUNEL staining in the GM of the spinal cord at $2 \mathrm{~mm}$ rostral from lesion site at $1 \mathrm{~d}$ and (D) in the WM at $7 \mathrm{~mm}$ rostral from lesion site at $5 \mathrm{~d}$. Bottom panels show high-power views $(\mathrm{n}=5)$. Scale bars, $20 \mu \mathrm{m}$. (E) Quantitative analysis of TUNEL-positive cells. (F) Immunostaining of cleaved (active) caspase-3 in the WM at $5 \mathrm{~d}$ after injury (n $=5$ ). Scale bar, $20 \mu \mathrm{m}$. (G) Immunohistochemical analysis of cleaved caspase-3 and CC1. Double labeling shows that oligodendrocytes in the WM were positive for cleaved caspse-3 after SCI (arrow). Scale bar, 30 $\mu \mathrm{m}$. (H), Quantitative analysis of cleaved caspase-3-positive cells. (I) Western blot and (J) densitometirc analysis of cleaved caspase- 3 at $1 \mathrm{~d}$ and $5 \mathrm{~d}$ after injury $(\mathrm{n}=5)$. All data represent mean $\pm \mathrm{SD} .{ }^{*} p<0.05$ vs. vehicle.

Figure 8. GA improves functional recovery after SCI. (A) BBB scores of vehicle and GA-treated groups after injury. (B) Grid walk test of vehicle and GA-treated groups at $35 \mathrm{~d}$ after injury. (C) Inclined plane test of vehicle- and GA-treated groups after injury. (D) Representative footprints obtained from each group at $35 \mathrm{~d}$ after SCI. All data are presented as mean \pm SEM $(\mathrm{n}=10) .{ }^{*} p<0.05$ vs. vehicle.

Figure 9. GA alleviates axon and myelin loss and decreases lesion volume after SCI. (A) Representative photographs of NF200-positive axons in spinal cords. Sections were selected $2 \mathrm{~mm}$ rostral to the lesion site. Note that GA treatment decreased the extent of axon loss after injury. Scale bars, $20 \mu \mathrm{m}$. (B) Quantitative analysis of NF200-positive axons in ventral (left) and dorsolateral (right) funiculi showed that the density of 
spared axons in the GA-treated group was significantly higher than that in the vehicle. NF200-positive axons were counted as described under Materials and Methods. (C) Representative photographs of 5-HT-positive axons in ventral horn areas in sections $3 \mathrm{~mm}$ caudal to the lesion site. Scale bars, $30 \mu \mathrm{m}$. (D) Transverse cryosections (lateral funiculus) selected from $2 \mathrm{~mm}$ rostral to the lesion site were processed for Luxol fast blue staining. Note that the extent of myelin loss was attenuated by GA treatment compared to the vehicle control. Scale bar, $100 \mu \mathrm{m}$. (E) Representative spinal cord tissues (1.2 mm from the dorsal surface) showing cavitation in the lesion site and quantitative analysis at $35 \mathrm{~d}$ after injury. Scale bar, $1 \mathrm{~mm}$. All data are presented as mean $\pm \mathrm{SD}(\mathrm{n}=5) .{ }^{*} p<0.05$ vs. vehicle.

\section{Hosted file}

Table_1.xlsx available at https://authorea.com/users/337067/articles/462732-gallic-acidattenuates-blood-spinal-cord-barrier-disruption-by-inhibiting-jmjd3-expression-andactivation-after-spinal-cord-injury

Experimental 1. Time course of sample preparation

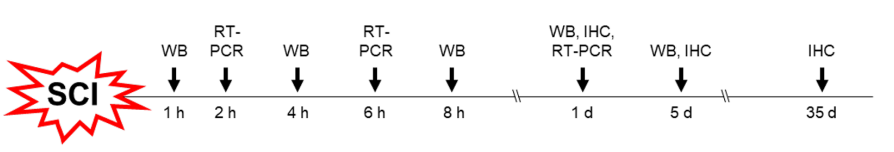

Group:

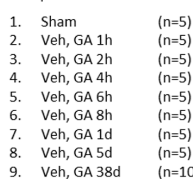

Experimental 2. Evans blue assay

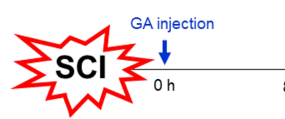

Experimental 3. Behavioral test

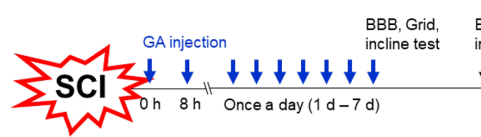

\section{BBB Grid BBB, Grid, BBid,}

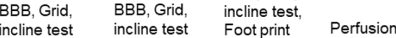

$\downarrow$

\section{Evans blue dye}

$24 \mathrm{~h}$
Group:

$\begin{array}{lll}\text { 1. Sham } & (n=5) \\ \text { 2. Veh } & (n=5) \\ \text { 3. GA } & (n=5)\end{array}$

Group:

$\begin{array}{lll}\text { 1. Sham } & (\mathrm{n}=10) \\ \text { 2. } & \text { Veh } & (\mathrm{n}=10) \\ \text { 3. } & \text { GA } & \end{array}$ 

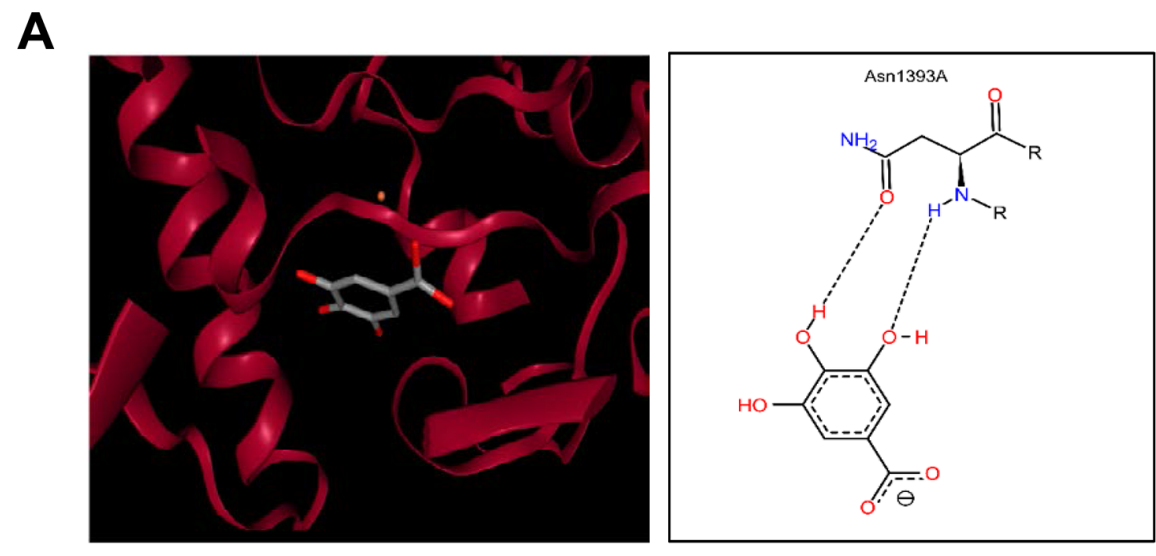

B

$$
\begin{array}{rccccl}
\text { Jmjd3 : } & - & + & + & + & + \\
\text { Inhibitor : } & - & - & \text { J4 } & \text { GA } & \text { GA } \\
\text { Conc : } & - & - & 2 & 2 & 10(\mu \mathrm{M})
\end{array}
$$
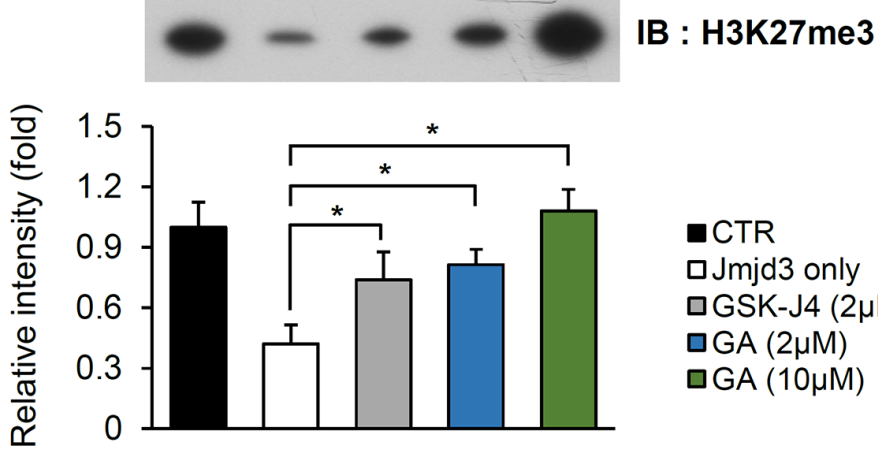

- CTR

口Jmjd3 only 口GSK-J4 $(2 \mu \mathrm{M})$ 口GA $(2 \mu \mathrm{M})$ 口GA $(10 \mu \mathrm{M})$ 
A
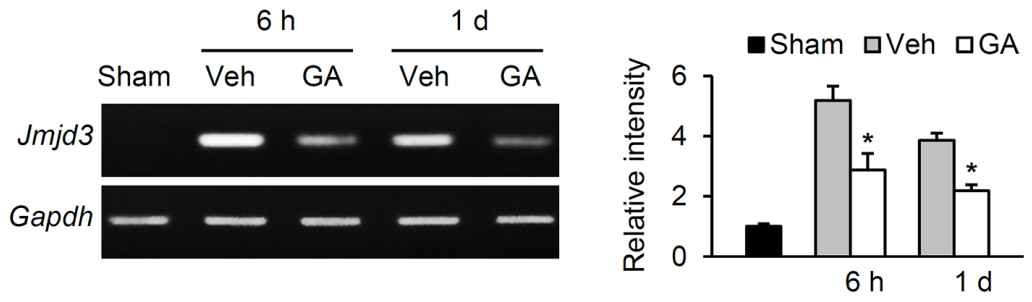

B
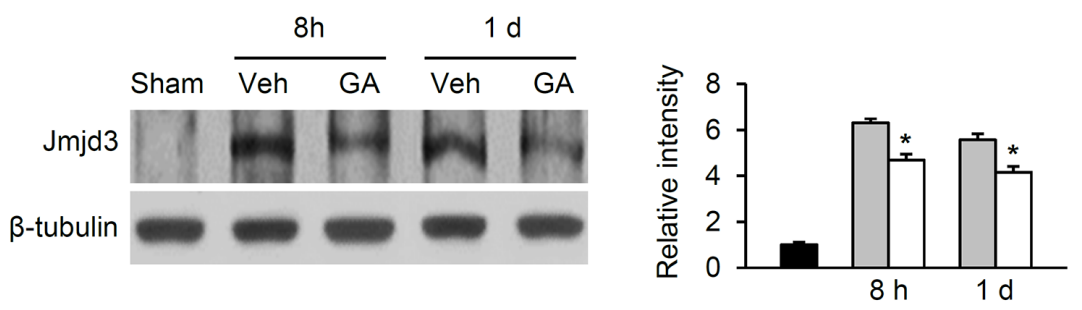

C
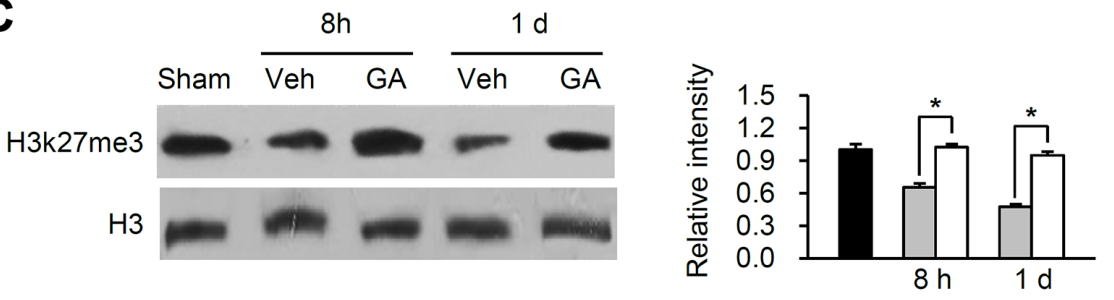

D
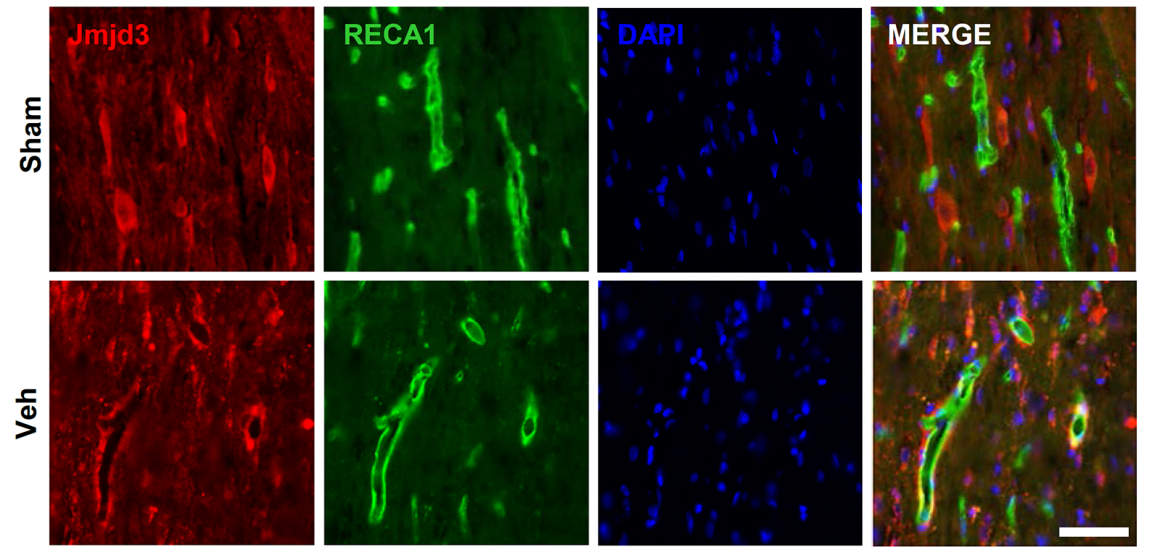
A
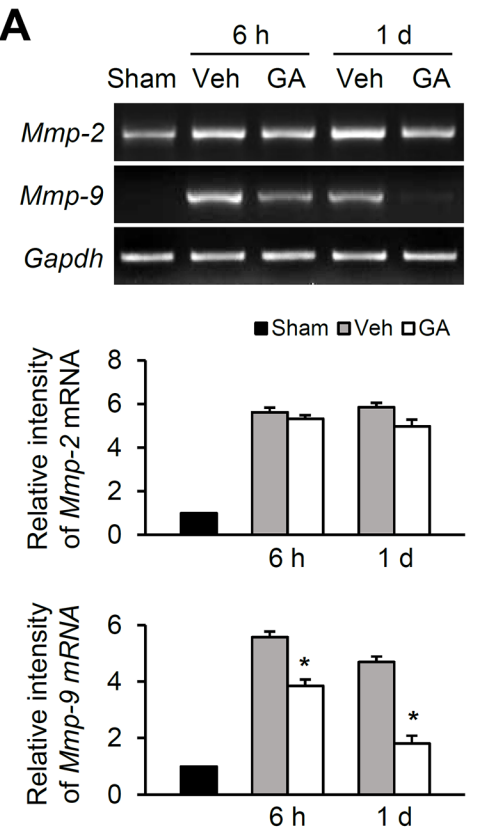

D

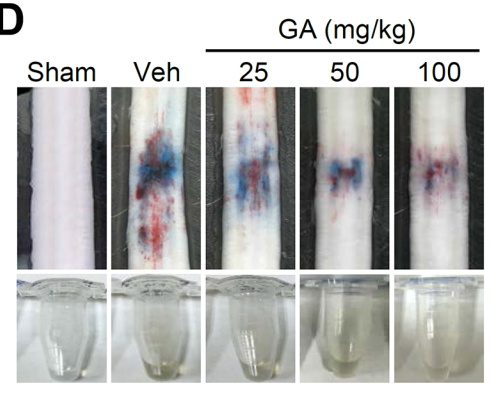

F

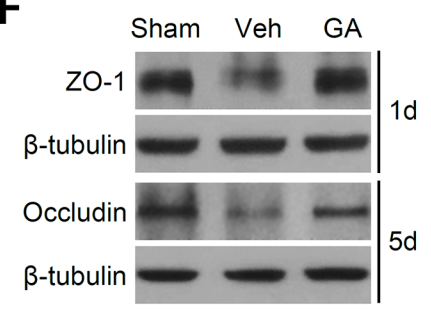

B

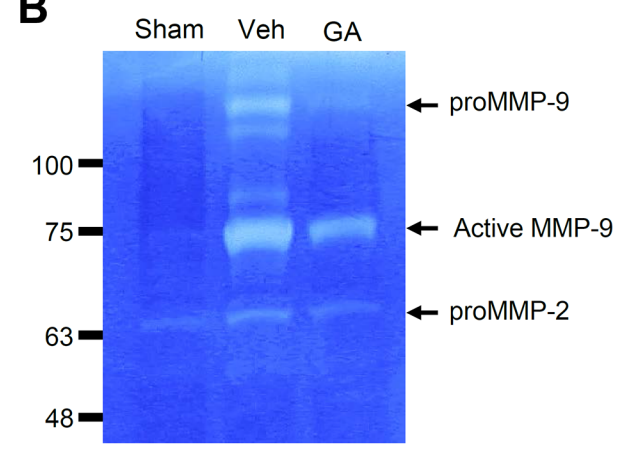

C

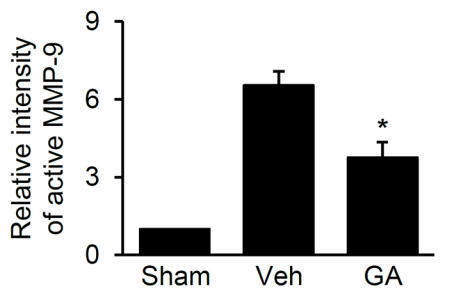

E
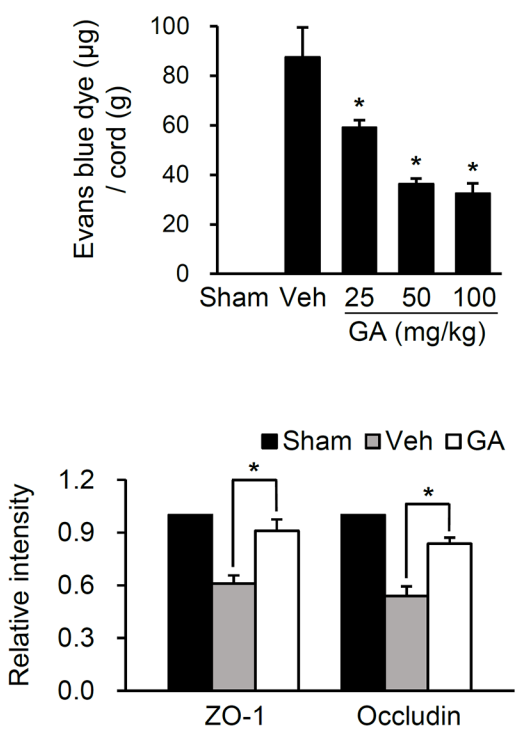
A

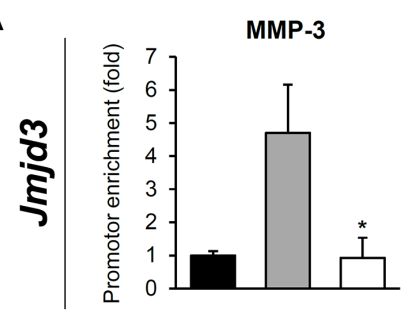

B
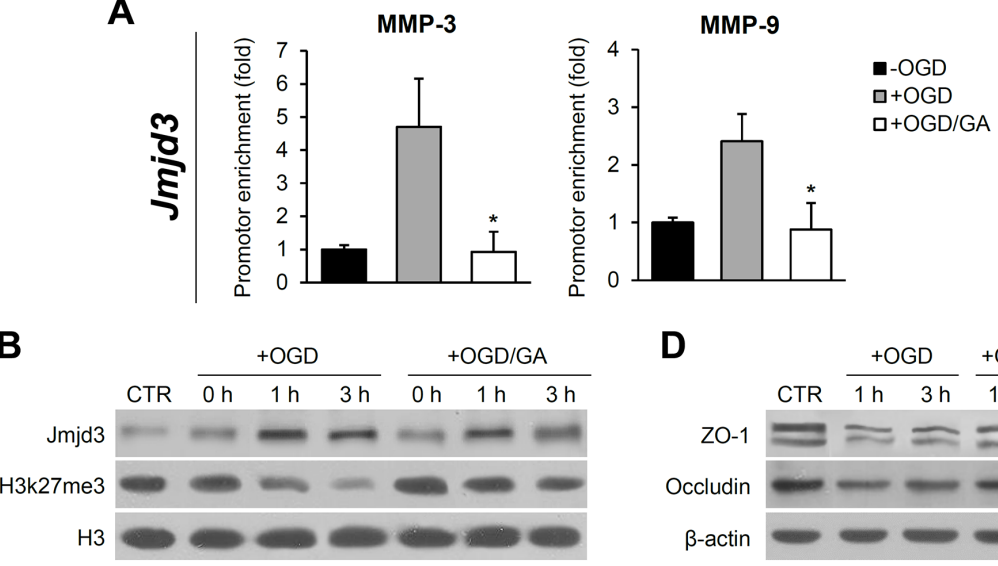

C

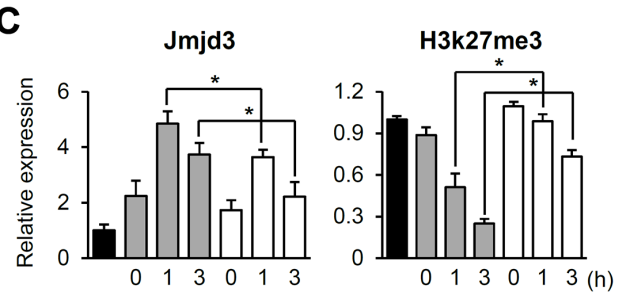

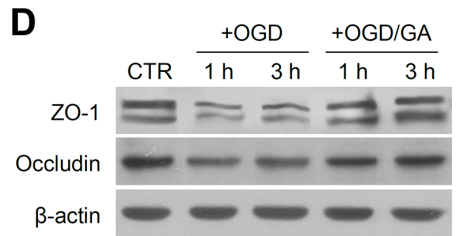

E

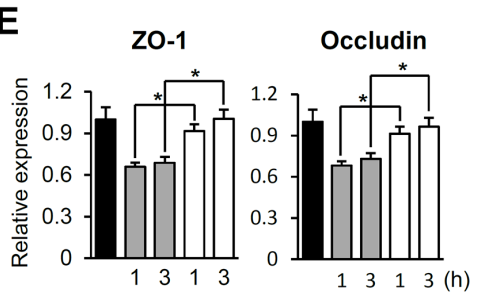

A
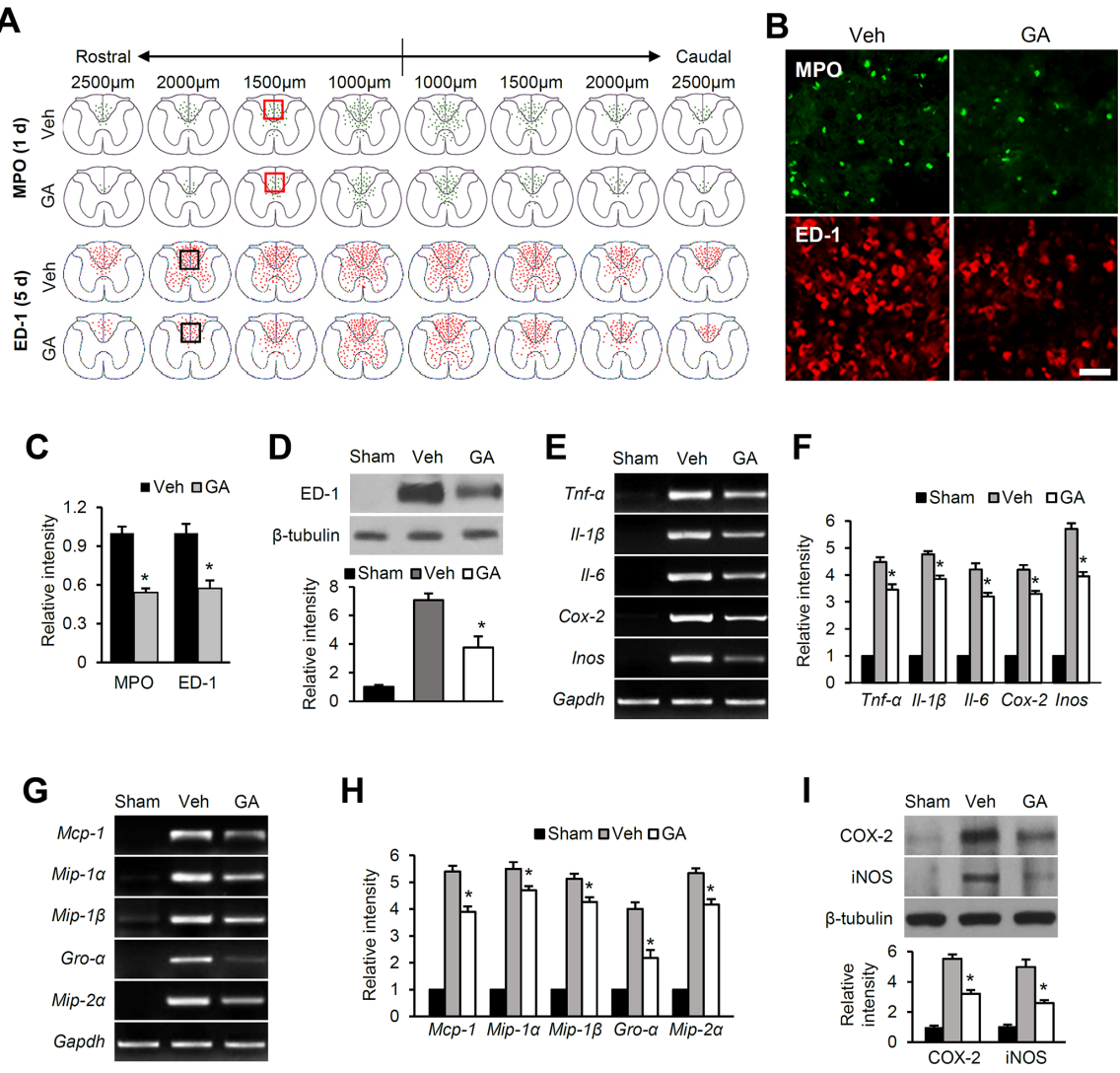
A

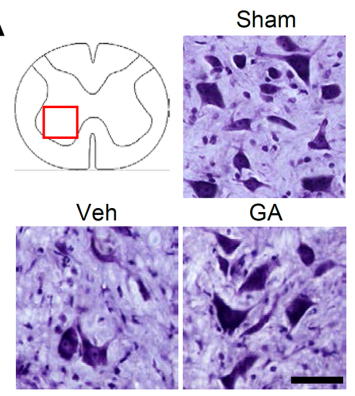

C

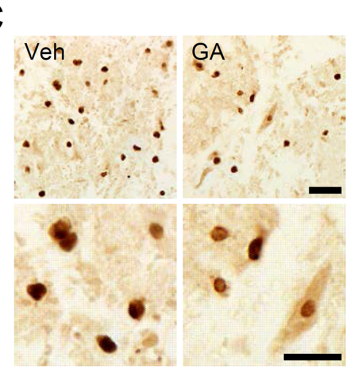

$\mathbf{F}$
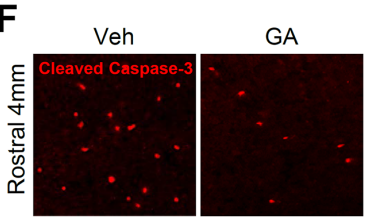

D

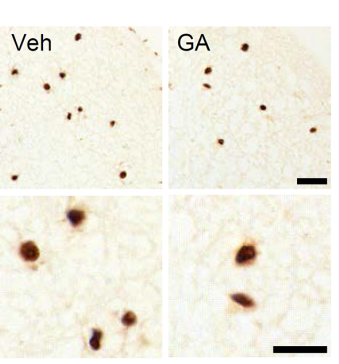

G
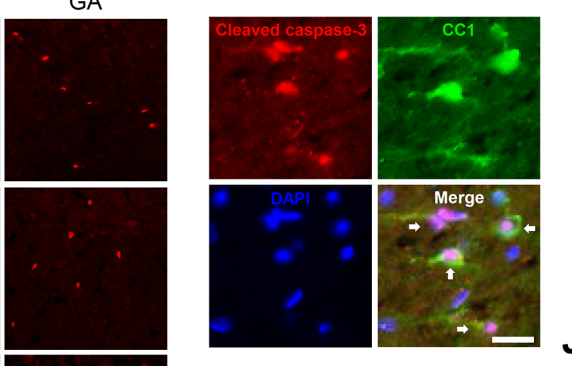

B

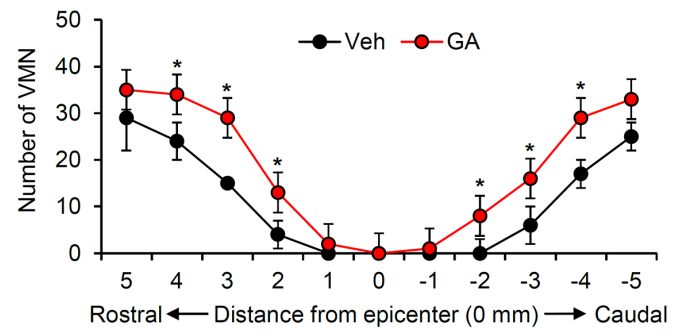

E

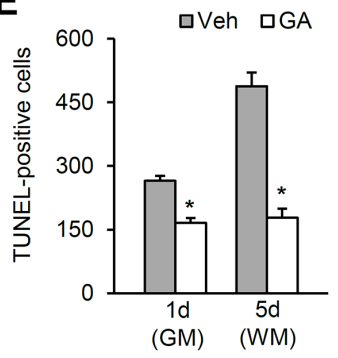

I

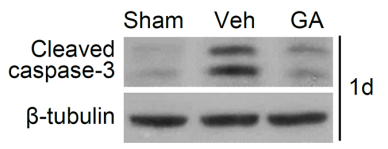

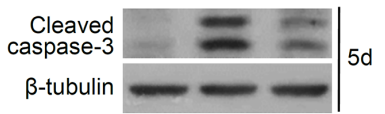

H

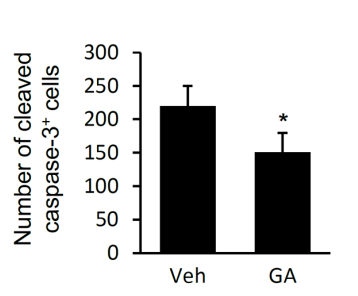

-Sham aveh DGA

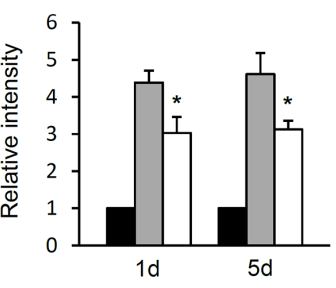



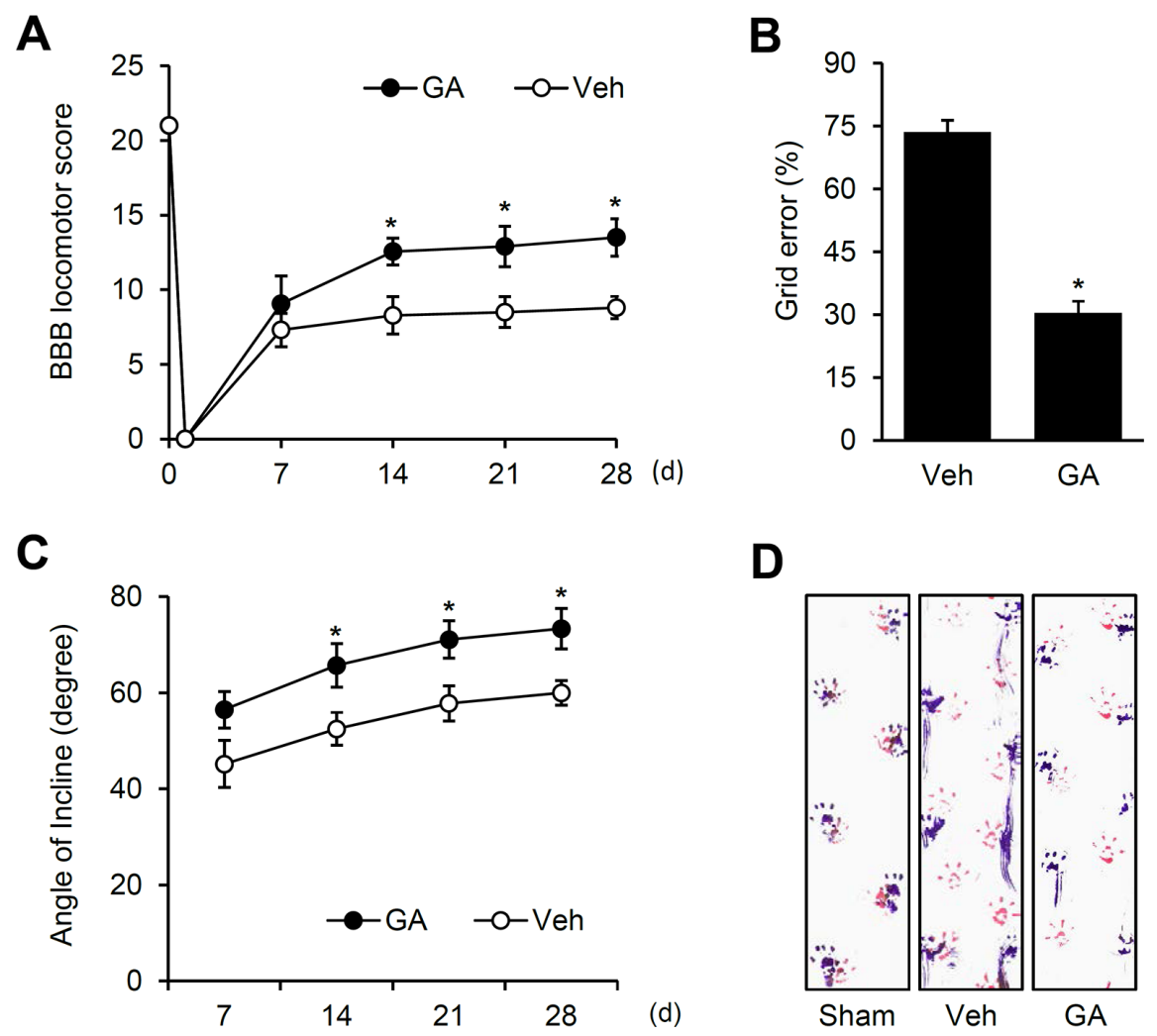

(d)

D

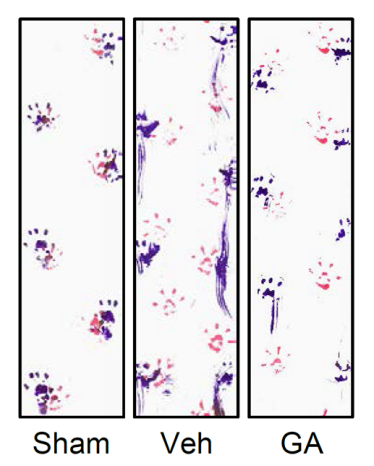


A
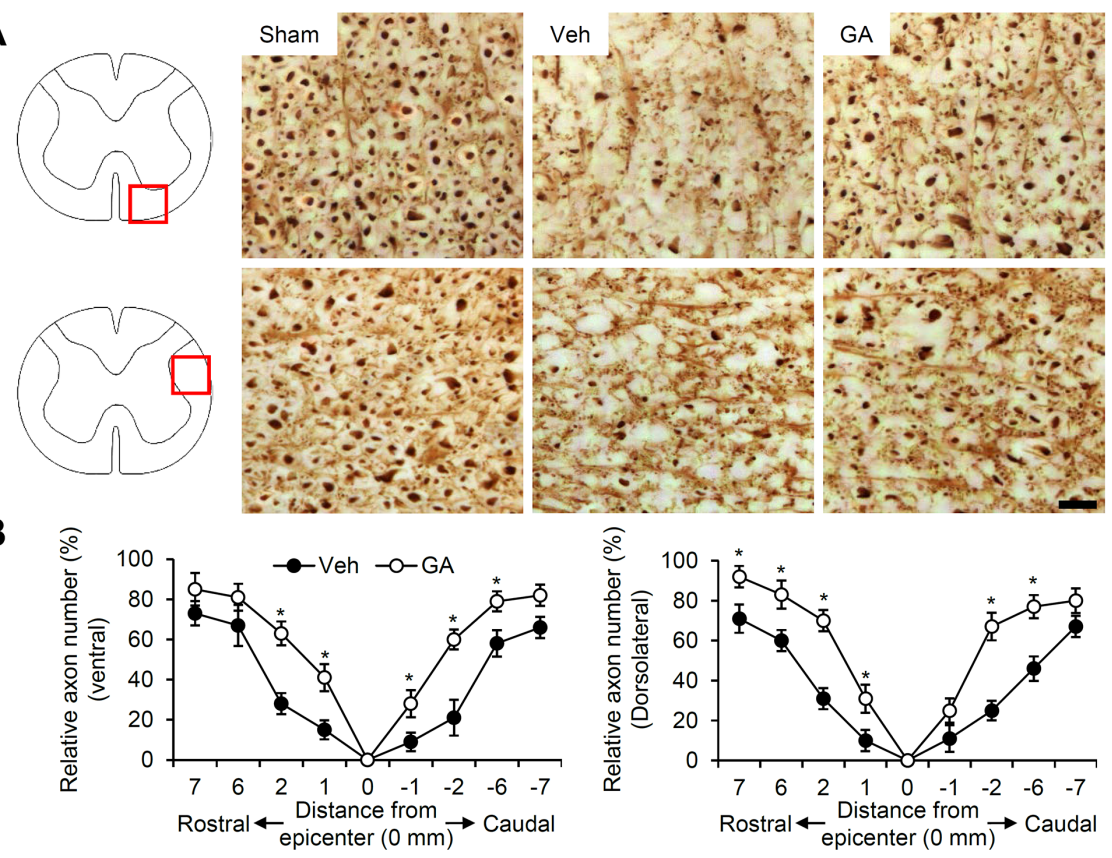

B

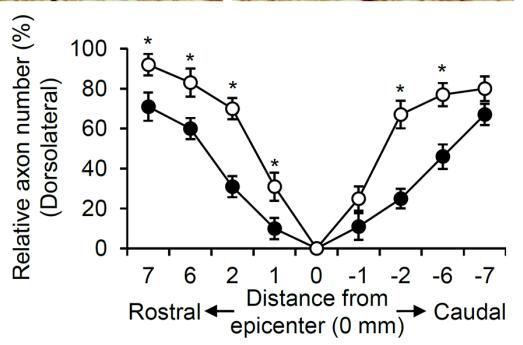

C

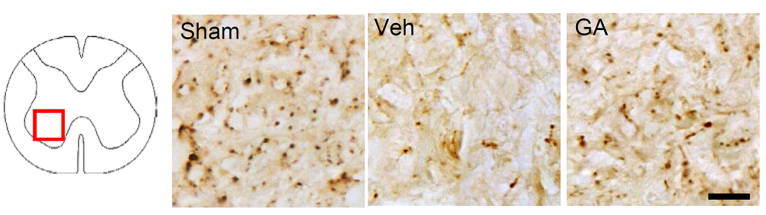

D

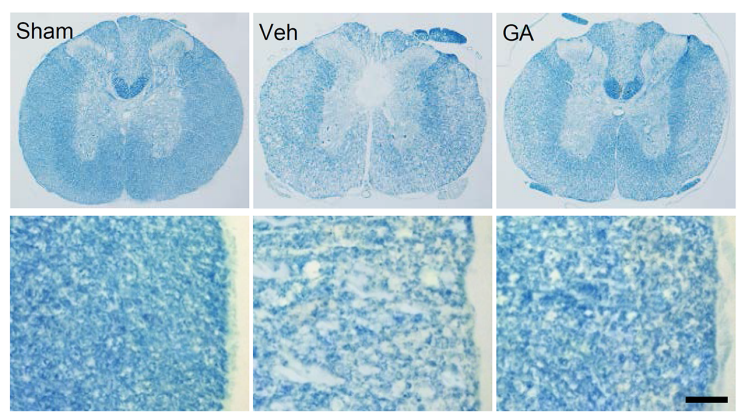

E
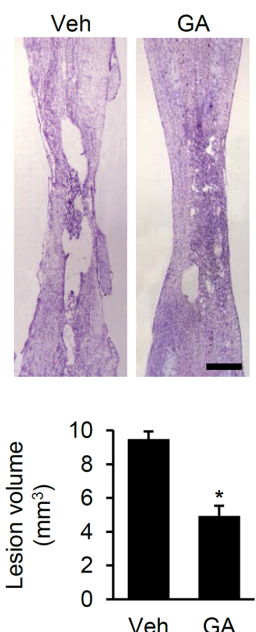